\title{
Analysis of the hidden-charm tetraquark mass spectrum with the QCD sum rules
}

\author{
Zhi-Gang Wang $\oplus^{*}$ \\ Department of Physics, North China Electric Power University, \\ Baoding 071003, People's Republic of China
}

(Received 15 October 2019; accepted 30 June 2020; published 13 July 2020)

\begin{abstract}
In this article, we take the pseudoscalar, scalar, axialvector, vector, tensor (anti)diquark operators as the basic constituents and construct the scalar, axialvector, and tensor tetraquark currents to study the mass spectrum of the ground state hidden-charm tetraquark states with the QCD sum rules in a comprehensive way. We revisit the assignments of the $X, Y, Z$ states, such as the $X(3860), X(3872), X(3915), X(3940)$, $X(4160), Z_{c}(3900), Z_{c}(4020), Z_{c}(4050), Z_{c}(4055), Z_{c}(4100), Z_{c}(4200), Z_{c}(4250), Z_{c}(4430), Z_{c}(4600)$, etc. in the scenario of tetraquark states in a consistent way based on the QCD sum rules. Furthermore, we discuss the feasibility of applying the QCD sum rules to study the tetraquark states and tetraquark molecular states (more precisely, the color-singlet-color-singlet type tetraquark states), which begin to receive contributions at the order $\mathcal{O}\left(\alpha_{s}^{0}\right)$, not at the order $\mathcal{O}\left(\alpha_{s}^{2}\right)$.
\end{abstract}

DOI: 10.1103/PhysRevD.102.014018

\section{INTRODUCTION}

In 2003, the Belle Collaboration observed a narrow charmoniumlike state $X(3872)$ in the $\pi^{+} \pi^{-} J / \psi$ mass spectrum in the exclusive decays $B^{ \pm} \rightarrow K^{ \pm} \pi^{+} \pi^{-} J / \psi$ [1], which cannot be accommodated in the conventional two quark model as the $\chi_{c 1}^{\prime}$ state with the quantum numbers $J^{P C}=1^{++}$. Thereafter, about twenty charmoniumlike states were observed by the BABAR, Belle, BESIII, CDF, CMS, D0, LHCb Collaborations [2], which cannot be accommodated in the conventional two quark model and are denoted as the $X, Y$, and $Z$ states now; some are still needing confirmation, and the quantum numbers have not been established yet. In Table I, we list out the masses, widths, and $J^{P C}$ of the $X, Y, Z$ states in the $\bar{c} c$ region from the Particle Data Group [2]. In 2018, the LHCb Collaboration observed evidence for an exotic $\eta_{c} \pi^{-}$resonant state [now referred to as $Z_{c}(4100)$ ] in the $B^{0} \rightarrow$ $\eta_{c} K^{+} \pi^{-}$decays with the significance of more than 3 standard deviations; the possible spin-parity assignments are $J^{P}=0^{+}$and $1^{-}$[3]. We add the $Z_{c}(4100)$ in Table I.

There have seen several possible interpretations for those $X, Y$, and $Z$ states, such as the tetraquark states, hadronic molecular states, dynamically generated resonances, hadroquarkonium, kinematical effects, cusp effects,

\footnotetext{
*zgwang@aliyun.com.
}

Published by the American Physical Society under the terms of the Creative Commons Attribution 4.0 International license. Further distribution of this work must maintain attribution to the author(s) and the published article's title, journal citation, and DOI. Funded by SCOAP ${ }^{3}$. virtual states, etc. using the phenomenological approaches (potential quark models), effective field theories for QCD (such as the heavy quark effective field theory, nonrelativistic QCD, potential nonrelativistic QCD, Born-Oppenheimer approximation, chiral unitary models), QCD sum rules, lattice QCD, etc. For comprehensive reviews, one can consult Refs. [4-12]. In the present work, we will focus on the tetraquark interpretations.

The QCD sum rules are a powerful theoretical approach in studying the hadron properties and have been applied extensively to calculate the masses, decay constants, form factors, hadronic coupling constants, etc. [13-15]. In 2006, R. D. Matheus et al. took the $X(3872)$ as the $J^{P C}=1^{++}$ diquark-antidiquark type tetraquark state and studied its mass with the QCD sum rules by carrying out the operator product expansion up to the vacuum condensates of dimension 8 [16]. Thereafter, the QCD sum rules became a powerful theoretical approach for studying the masses and widths of the $X, Y$, and $Z$ states, irrespective of the hidden-charm (or hidden-bottom) tetraquark states or hadronic molecular states [10,16-25]. In the QCD sum rules, we choose the color-antitriplet-color-triplet $\left(\overline{\mathbf{3}_{c}} \mathbf{3}_{c}\right)$ type, in other words, the diquark-antidiquark type, colorsextet-color-antisextet $\quad\left(\mathbf{6}_{c} \overline{\mathbf{6}}_{c}\right)$ type, color-singlet-colorsinglet $\left(\mathbf{1}_{c} \mathbf{1}_{c}\right)$ type, and color-octet-color-octet $\left(\mathbf{8}_{c} \mathbf{8}_{c}\right)$ type local four-quark currents to study the tetraquark states. It is better to call the corresponding tetraquark states as the $\overline{\mathbf{3}_{c}} \mathbf{3}_{c}$-type, $\mathbf{6}_{c} \overline{\mathbf{6}}_{c}$-type, $\mathbf{1}_{c} \mathbf{1}_{c}$-type, and $\mathbf{8}_{c} \mathbf{8}_{c}$-type tetraquark states, respectively. In the literatures, we usually call the $\overline{\mathbf{3}_{c}} \mathbf{3}_{c}$-type and $\mathbf{1}_{c} \mathbf{1}_{c}$-type tetraquark states as the tetraquark states and (tetraquark or hadronic) molecular states, respectively. Thereafter, we will use the name $\mathbf{1}_{c} \mathbf{1}_{c}$-type 
TABLE I. The masses, widths, and $J^{P C}$ of the $X, Y$, and $Z$ states in the $\bar{c} c$ region from the Particle Data Group except for the $Z_{c}(4100)$.

\begin{tabular}{|c|c|c|c|c|c|}
\hline State & $\mathrm{M}(\mathrm{MeV})$ & $\Gamma(\mathrm{MeV})$ & $J^{P C}$ & Process & Experiment \\
\hline$X(3860)$ & $3862_{-32}^{+26}{ }_{-13}^{+40}$ & $201_{-67}^{+154}{ }_{-82}^{+88}$ & $0^{++}$ & $e^{+} e^{-} \rightarrow J / \psi D \bar{D}$ & Belle \\
\hline$X(3872)$ & $3871.69 \pm 0.17$ & $<1.2$ & $1^{++}$ & $B \rightarrow K \pi^{+} \pi^{-} J / \psi$ & Belle \\
\hline$X(3915)$ & $3918.4 \pm 1.9$ & $20 \pm 5$ & $0 / 2^{++}$ & $B \rightarrow K J / \psi \omega$ & Belle, $B A B A R$ \\
\hline$X(3940)$ & $3942_{-6}^{+7} \pm 6$ & $37_{-15}^{+26} \pm 8$ & $? ?$ & $e^{+} e^{-} \rightarrow J / \psi D \bar{D}^{*}$ & Belle \\
\hline$X(4140)$ & $4146.8 \pm 2.4$ & $22_{-7}^{+8}$ & $1^{++}$ & $B^{+} \rightarrow J / \psi \phi K^{+}$ & $\mathrm{CDF}, \mathrm{D} 0, \mathrm{LHCb}$ \\
\hline$X(4160)$ & $4156_{-20}^{+25} \pm 15$ & $139_{-61}^{+111} \pm 21$ & $? ? ?$ & $e^{+} e^{-} \rightarrow J / \psi D^{*} \bar{D}^{*}$ & Belle \\
\hline$X(4274)$ & $4274_{-6}^{+8}$ & $49 \pm 12$ & $1^{++}$ & $B^{+} \rightarrow J / \psi \phi K^{+}$ & $\mathrm{CDF}, \mathrm{LHCb}$ \\
\hline$X(4350)$ & $4350.6_{-5.1}^{+4.6} \pm 0.7$ & $13_{-9}^{+18} \pm 4$ & $?++$ & $e^{+} e^{-} \rightarrow \phi J / \psi$ & Belle \\
\hline$X(4500)$ & $4506 \pm 11_{-15}^{+12}$ & $92 \pm 21_{-20}^{+21}$ & $0^{++}$ & $B^{+} \rightarrow J / \psi \phi K^{+}$ & $\mathrm{LHCb}$ \\
\hline$X(4700)$ & $4704 \pm 10_{-24}^{+14}$ & $120 \pm 31_{-33}^{+42}$ & $0^{++}$ & $B^{+} \rightarrow J / \psi \phi K^{+}$ & $\mathrm{LHCb}$ \\
\hline$Y(4220)$ & $4218_{-4}^{+5}$ & $59_{-10}^{+12}$ & $1^{--}$ & $e^{+} e^{-} \rightarrow h_{c} \pi^{+} \pi^{-}$ & BESIII \\
\hline$Y(4360)$ & $4368 \pm 13$ & $96 \pm 7$ & $1^{--}$ & $e^{+} e^{-} \rightarrow \pi^{+} \pi^{-} \psi^{\prime}$ & $B A B A R$, Belle \\
\hline$Y(4390)$ & $4391.5_{-6.8}^{+6.3} \pm 1.0$ & $139.5_{-20.6}^{+16.2} \pm 0.6$ & $1^{--}$ & $e^{+} e^{-} \rightarrow h_{c} \pi^{+} \pi^{-}$ & BESIII \\
\hline$Y(4660)$ & $4643 \pm 9$ & $72 \pm 11$ & $1^{--}$ & $e^{+} e^{-} \rightarrow \pi^{+} \pi^{-} \psi^{\prime}$ & Belle, $B A B A R$ \\
\hline$Z_{c}(3900)$ & $3887.2 \pm 2.3$ & $28.2 \pm 2.6$ & $1^{+-}$ & $Y(4260) \rightarrow J / \psi \pi^{+} \pi^{-}$ & BESIII, Belle \\
\hline$Z_{c}(4020)$ & $4024.1 \pm 1.9$ & $13 \pm 5$ & $?^{?-}$ & $e^{+} e^{-} \rightarrow \pi^{-} \pi^{+} h_{c}$ & BESIII \\
\hline$Z_{c}(4050)$ & $4051 \pm 14_{-41}^{+20}$ & $82_{-17-22}^{+21+47}$ & $?^{?+}$ & $\bar{B}^{0} \rightarrow K^{-} \pi^{+} \chi_{c 1}$ & Belle \\
\hline$Z_{c}(4055)$ & $4054 \pm 3 \pm 1$ & $45 \pm 11 \pm 6$ & $?^{?-}$ & $e^{+} e^{-} \rightarrow \pi^{-} \pi^{+} \psi^{\prime}$ & Belle \\
\hline$Z_{c}(4100)$ & $4096 \pm 20_{-22}^{+18}$ & $152 \pm 58_{-35}^{+60}$ & $0^{++} / 1^{-+}$ & $B^{0} \rightarrow K^{+} \pi^{-} \eta_{c}$ & $\mathrm{LHCb}$ \\
\hline$Z_{c}(4200)$ & $4196_{-29}^{+31+13}$ & $370 \pm 70_{-132}^{+70}$ & $1^{+-}$ & $\bar{B}^{0} \rightarrow K^{-} \pi^{+} J / \psi$ & Belle \\
\hline$Z_{c}(4250)$ & $4248_{-29}^{+44+180}$ & $\begin{array}{l}177_{-39-61}^{+54}+316 \\
-{ }_{-6}\end{array}$ & $?++$ & $\bar{B}^{0} \rightarrow K^{-} \pi^{+} \chi_{c 1}$ & Belle \\
\hline$Z_{c}(4430)$ & $4478_{-18}^{+15}$ & $181 \pm 31$ & $1^{+-}$ & $B \rightarrow K^{-} \pi^{+} \psi^{\prime}$ & Belle, LHCb \\
\hline
\end{tabular}

tetraquark states instead of the name (tetraquark or hadronic) molecular states according to the local currents.

In the QCD sum rules for the hidden-charm (or hiddenbottom) tetraquark states and $\mathbf{1}_{c} \mathbf{1}_{c}$-type tetraquark states, the integrals,

$$
\int_{4 m_{Q}^{2}(\mu)}^{s_{0}} d s \rho_{\mathrm{QCD}}(s, \mu) \exp \left(-\frac{s}{T^{2}}\right),
$$

are sensitive to the heavy quark masses $m_{Q}(\mu)$; more precisely speaking, the integrals are sensitive to the energy scales $\mu$, where the $\rho_{\mathrm{QCD}}(s, \mu)$ are the QCD spectral densities, the $T^{2}$ are the Borel parameters, and the $s_{0}$ are the continuum threshold parameters. In Ref. [17], we tentatively assign $X(3872)$ and $Z_{c}(3900)$ to be the diquarkantidiquark type axialvector tetraquark states, and study them with the QCD sum rules in detail, and explore the energy scale dependence of the QCD sum rules for the hidden-charm tetraquark states for the first time [17]. In Ref. [19], we suggest a formula,

$$
\mu=\sqrt{M_{X / Y / Z}^{2}-\left(2 M_{c}\right)^{2}}
$$

with the effective heavy mass $\mathbb{M}_{c}$ to determine the optimal energy scales, which can be applied to study the hidden-bottom tetraquark states directly with the simple replacement $\mathbb{M}_{c} \rightarrow \mathbb{M}_{b}[20]$.

In the scenario of tetraquark states, the $Y$ states, i.e., the $Y(4220), Y(4360), Y(4390), Y(4660)$, can be assigned to be the diquark-antidiquark type tetraquark states. In Ref. [21], we introduce a relative $\mathrm{P}$ wave between the diquark and antidiquark operators explicitly for constructing the tetraquark currents to systematically study the vector tetraquark states with the QCD sum rules and obtain the lowest vector tetraquark masses up to now, which support assigning the $Y(4220 / 4260), Y(4320 / 4360)$, $Y(4390)$, and $Z_{c}(4250)$ to be the vector hidden-charm tetraquark states. While novel analysis of the masses and widths of the vector hidden-charm tetraquark states without a relative $\mathrm{P}$ wave between the diquark and antidiquark constituents indicate that the $Y(4660)$ can be assigned to be a $[s c]_{P}[\bar{s} \bar{c}]_{A}-[s c]_{A}[\bar{s} \bar{c}]_{P}$ type tetraquark state [22], in those studies, the energy scale formula and modified energy scale formula play an important role in enhancing the pole contributions and in improving the convergent behavior of the operator product expansion.

The $X$ states $X(4140), X(4274), X(4350), X(4500)$, and $X(4700)$ are observed in the $J / \psi \phi$ mass spectrum; if their dominant Fock components are tetraquark states, their quark constituents are $\bar{c} c \bar{s} s$ rather than $\bar{c} c \bar{q} q$. The QCD sum rules support assigning the $X(3915), X(4140), X(4274)$, $X(4500)$, and $X(4700)$ to be the diquark-antidiquark type 
tetraquark states [26,27]; the decay $X(3915) \rightarrow J / \psi \phi \rightarrow$ $J / \psi \omega$ can take place through the $\phi-\omega$ mixing [28].

The $X$ states $X(3860), X(3872), X(3940)$, and $X(4160)$ are observed in the final states $D \bar{D}, D \bar{D}^{*}, D^{*} \bar{D}, D^{*} \bar{D}^{*}$, or $J / \psi \pi \pi$; if their dominant Fock components are tetraquark states, their constituents are $\bar{c} c \bar{q} q$.

The $Z$ states $Z_{c}(3900), Z_{c}(4020), Z_{c}(4050), Z_{c}(4055)$, $Z_{c}(4100), Z_{c}(4200), Z_{c}(4250)$, and $Z_{c}(4430)$ have a nonzero electric charge, which prevent them from being the conventional two-quark mesons; they are excellent candidates for the tetraquark states. Those $Z_{c}$ states have been studied with the QCD sum rules in one way or another $[10,17,19,23,24,29-31]$.

We usually take the diquarks in color antitriplet $\overline{\mathbf{3}}_{c}$ as the basic building blocks to construct the tetraquark states. The diquarks operators $\varepsilon^{a b c} q_{b}^{T} C \Gamma q_{c}^{\prime}$ have five structures in Dirac spinor space, where $C \Gamma=C \gamma_{5}, C, C \gamma_{\mu} \gamma_{5}, C \gamma_{\mu}$, and $C \sigma_{\mu \nu}$ (or $C \sigma_{\mu \nu} \gamma_{5}$ ) for the scalar $(S)$, pseudoscalar $(P)$, vector $(V)$, axialvector $(A)$, and tensor $(T)$ diquarks, respectively; the $a, b, c$ are color indexes. The tensor diquark states have both $J^{P}=1^{+}$and $1^{-}$components; we project out the $1^{+}$ and $1^{-}$components explicitly and denote the corresponding axialvector and vector diquarks as $\tilde{A}$ and $\tilde{V}$, respectively.

All in all, those $X, Y$, and $Z$ states have been studied with the QCD sum rules in one way or another [10,16-27, 29-31]; in the present work, we take the scalar, pseudoscalar, axialvector, vector, and tensor diquark operators as the basic building blocks to construct twenty tetraquark currents and study the mass spectrum of the hidden-charm tetraquark states with the QCD sum rules in a comprehensive way and revisit the assignments of the $X$ and $Z$ states in the scenario of tetraquark states and try to accommodate the exotic states as many as possible in a consistent way. We take the energy scale formula $\mu=\sqrt{M_{X / Y / Z}^{2}-\left(2 \mathbb{M}_{c}\right)^{2}}$ to determine the best energy scales of the QCD spectral densities so as to enhance the pole contributions and improve the convergent behaviors of the operator product expansion [19]. In other words, the predicted hidden-charm tetraquark masses and the QCD spectral densities satisfy the relation $M_{X / Y / Z}^{2}=\mu^{2}+4 \mathbb{M}_{c}^{2}$, where the $\mathbb{M}_{c}$ has an universal value. It is an unique feature of our works. We obtain new results for eleven tetraquark currents and present them here for the first time; for other tetraquark currents, we recalculate the vacuum condensates up to dimension 10 in the operator product expansion consistently and preform updated analysis [17,29,31]. Those hidden-charm tetraquark states may be observed at the BESIII, LHCb, Belle II, CEPC, FCC, ILC in the future and shed light on the nature of the exotic $X, Y, Z$ states.

The article is arranged as follows: we derive the QCD sum rules for the masses and pole residues of the hiddencharm tetraquark states in Sec. II; in Sec. III, we present the numerical results and discussions; Sec. IV is reserved for our conclusion.

\section{QCD SUM RULES FOR THE HIDDEN-CHARM TETRAQUARK STATES}

We write down the two-point correlation functions $\Pi(p)$, $\Pi_{\mu \nu}(p)$ and $\Pi_{\mu \nu \alpha \beta}(p)$ in the QCD sum rules,

$$
\begin{aligned}
\Pi(p) & =i \int d^{4} x e^{i p \cdot x}\left\langle 0\left|T\left\{J(x) J^{\dagger}(0)\right\}\right| 0\right\rangle, \\
\Pi_{\mu \nu}(p) & =i \int d^{4} x e^{i p \cdot x}\left\langle 0\left|T\left\{J_{\mu}(x) J_{\nu}^{\dagger}(0)\right\}\right| 0\right\rangle, \\
\Pi_{\mu \nu \alpha \beta}(p) & =i \int d^{4} x e^{i p \cdot x}\left\langle 0\left|T\left\{J_{\mu \nu}(x) J_{\alpha \beta}^{\dagger}(0)\right\}\right| 0\right\rangle,
\end{aligned}
$$

where the currents $J(x)=J_{S S}(x), J_{A A}(x), J_{\tilde{A} \tilde{A}}(x), J_{V V}(x)$, $J_{\tilde{V} \tilde{V}}(x), J_{P P}(x), J_{\mu}(x)=J_{-, \mu}^{S A}(x), J_{-, \mu}^{\tilde{A} A}(x), J_{-, \mu}^{\tilde{V} V}(x), J_{-, \mu}^{P V}(x)$, $J_{+, \mu}^{S A}(x), \quad J_{+, \mu}^{\tilde{V} V}(x), \quad J_{+, \mu}^{\tilde{A} A}(x), \quad J_{+, \mu}^{P V}(x), \quad J_{\mu \nu}(x)=J_{-, \mu \nu}^{A A}(x)$, $J_{-, \mu \nu}^{S \tilde{A}}(x), J_{-, \mu \nu}^{V V}(x), J_{+, \mu \nu}^{S \tilde{A}}(x), J_{+, \mu \nu}^{A A}(x), J_{+, \mu \nu}^{V V}(x)$,

$$
\begin{aligned}
J_{S S}(x) & =\varepsilon^{i j k} \varepsilon^{i m n} u^{T j}(x) C \gamma_{5} c^{k}(x) \bar{d}^{m}(x) \gamma_{5} C \bar{c}^{T n}(x), \\
J_{A A}(x) & =\varepsilon^{i j k} \varepsilon^{i m n} u^{T j}(x) C \gamma_{\mu} c^{k}(x) \bar{d}^{m}(x) \gamma^{\mu} C \bar{c}^{T n}(x), \\
J_{\tilde{A} \tilde{A}}(x) & =\varepsilon^{i j k} \varepsilon^{i m n} u^{T j}(x) C \sigma_{\mu \nu}^{v} c^{k}(x) \bar{d}^{m}(x) \sigma_{v}^{\mu \nu} C \bar{c}^{T n}(x), \\
J_{V V}(x) & =\varepsilon^{i j k} \varepsilon^{i m n} u^{T j}(x) C \gamma_{\mu} \gamma_{5} c^{k}(x) \bar{d}^{m}(x) \gamma_{5} \gamma^{\mu} C \bar{c}^{T n}(x), \\
J_{\tilde{V} \tilde{V}}(x) & =\varepsilon^{i j k} \varepsilon^{i m n} u^{T j}(x) C \sigma_{\mu \nu}^{t} c^{k}(x) \bar{d}^{m}(x) \sigma_{t}^{\mu \nu} C \bar{c}^{T n}(x), \\
J_{P P}(x) & =\varepsilon^{i j k} \varepsilon^{i m n} u^{T j}(x) C c^{k}(x) \bar{d}^{m}(x) C \bar{c}^{T n}(x),
\end{aligned}
$$

$$
\begin{aligned}
& J_{-, \mu}^{S A}(x)=\frac{\varepsilon^{i j k} \varepsilon^{i m n}}{\sqrt{2}}\left[u^{T j}(x) C \gamma_{5} c^{k}(x) \bar{d}^{m}(x) \gamma_{\mu} C \bar{c}^{T n}(x)-u^{T j}(x) C \gamma_{\mu} c^{k}(x) \bar{d}^{m}(x) \gamma_{5} C \bar{c}^{T n}(x)\right], \\
& J_{-, \mu \nu}^{A A}(x)=\frac{\varepsilon^{i j k} \varepsilon^{i m n}}{\sqrt{2}}\left[u^{T j}(x) C \gamma_{\mu} c^{k}(x) \bar{d}^{m}(x) \gamma_{\nu} C \bar{c}^{T n}(x)-u^{T j}(x) C \gamma_{\nu} c^{k}(x) \bar{d}^{m}(x) \gamma_{\mu} C \bar{c}^{T n}(x)\right], \\
& J_{-, \mu \nu}^{S \tilde{A}}(x)=\frac{\varepsilon^{i j k} \varepsilon^{i m n}}{\sqrt{2}}\left[u^{T j}(x) C \gamma_{5} c^{k}(x) \bar{d}^{m}(x) \sigma_{\mu \nu} C \bar{c}^{T n}(x)-u^{T j}(x) C \sigma_{\mu \nu} c^{k}(x) \bar{d}^{m}(x) \gamma_{5} C \bar{c}^{T n}(x)\right], \\
& J_{-, \mu}^{\tilde{A} A}(x)=\frac{\varepsilon^{i j k} \varepsilon^{i m n}}{\sqrt{2}}\left[u^{T j}(x) C \sigma_{\mu \nu} \gamma_{5} c^{k}(x) \bar{d}^{m}(x) \gamma^{\nu} C \bar{c}^{T n}(x)-u^{T j}(x) C \gamma^{\nu} c^{k}(x) \bar{d}^{m}(x) \gamma_{5} \sigma_{\mu \nu} C \bar{c}^{T n}(x)\right],
\end{aligned}
$$




$$
\begin{aligned}
J_{-, \mu}^{\tilde{V} V}(x) & =\frac{\varepsilon^{i j k} \varepsilon^{i m n}}{\sqrt{2}}\left[u^{T j}(x) C \sigma_{\mu \nu} c^{k}(x) \bar{d}^{m}(x) \gamma_{5} \gamma^{\nu} C \bar{c}^{T n}(x)+u^{T j}(x) C \gamma^{\nu} \gamma_{5} c^{k}(x) \bar{d}^{m}(x) \sigma_{\mu \nu} C \bar{c}^{T n}(x)\right], \\
J_{-, \mu \nu}^{V V}(x)= & \frac{\varepsilon^{i j k} \varepsilon^{i m n}}{\sqrt{2}}\left[u^{T j}(x) C \gamma_{\mu} \gamma_{5} c^{k}(x) \bar{d}^{m}(x) \gamma_{5} \gamma_{\nu} C \bar{c}^{T n}(x)-u^{T j}(x) C \gamma_{\nu} \gamma_{5} c^{k}(x) \bar{d}^{m}(x) \gamma_{5} \gamma_{\mu} C \bar{c}^{T n}(x)\right], \\
J_{-, \mu}^{P V}(x)= & \frac{\varepsilon^{i j k} \varepsilon^{i m n}}{\sqrt{2}}\left[u^{T j}(x) C c^{k}(x) \bar{d}^{m}(x) \gamma_{5} \gamma_{\mu} C \bar{c}^{T n}(x)+u^{T j}(x) C \gamma_{\mu} \gamma_{5} c^{k}(x) \bar{d}^{m}(x) C \bar{c}^{T n}(x)\right], \\
J_{+, \mu}^{S A}(x)= & \frac{\varepsilon^{i j k} \varepsilon^{i m n}}{\sqrt{2}}\left[u^{T j}(x) C \gamma_{5} c^{k}(x) \bar{d}^{m}(x) \gamma_{\mu} C \bar{c}^{T n}(x)+u^{T j}(x) C \gamma_{\mu} c^{k}(x) \bar{d}^{m}(x) \gamma_{5} C \bar{c}^{T n}(x)\right], \\
J_{+, \mu \nu}^{S \tilde{A}}(x)= & \frac{\varepsilon^{i j k} \varepsilon^{i m n}}{\sqrt{2}}\left[u^{T j}(x) C \gamma_{5} c^{k}(x) \bar{d}^{m}(x) \sigma_{\mu \nu} C \bar{c}^{T n}(x)+u^{T j}(x) C \sigma_{\mu \nu} c^{k}(x) \bar{d}^{m}(x) \gamma_{5} C \bar{c}^{T n}(x)\right], \\
J_{+, \mu}^{\tilde{V} V}(x)= & \frac{\varepsilon^{i j k} \varepsilon^{i m n}}{\sqrt{2}}\left[u^{T j}(x) C \sigma_{\mu \nu} c^{k}(x) \bar{d}^{m}(x) \gamma_{5} \gamma^{\nu} C \bar{c}^{T n}(x)-u^{T j}(x) C \gamma^{\nu} \gamma_{5} c^{k}(x) \bar{d}^{m}(x) \sigma_{\mu \nu} C \bar{c}^{T n}(x)\right], \\
J_{+, \mu}^{\tilde{A} A}(x)= & \frac{\varepsilon^{i j k} \varepsilon^{i m n}}{\sqrt{2}}\left[u^{T j}(x) C \sigma_{\mu \nu} \gamma_{5} c^{k}(x) \bar{d}^{m}(x) \gamma^{\nu} C \bar{c}^{T n}(x)+u^{T j}(x) C \gamma^{\nu} c^{k}(x) \bar{d}^{m}(x) \gamma_{5} \sigma_{\mu \nu} C \bar{c}^{T n}(x)\right], \\
J_{+, \mu}^{P V}(x)= & \frac{\varepsilon^{i j k} \varepsilon^{i m n}}{\sqrt{2}}\left[u^{T j}(x) C c^{k}(x) \bar{d}^{m}(x) \gamma_{5} \gamma_{\mu} C \bar{c}^{T n}(x)-u^{T j}(x) C \gamma_{\mu} \gamma_{5} c^{k}(x) \bar{d}^{m}(x) C \bar{c}^{T n}(x)\right], \\
J_{+, \mu \nu}^{A A}(x)= & \frac{\varepsilon^{i j k} \varepsilon^{i m n}}{\sqrt{2}}\left[u^{T j}(x) C \gamma_{\mu} c^{k}(x) \bar{d}^{m}(x) \gamma_{\nu} C \bar{c}^{T n}(x)+u^{T j}(x) C \gamma_{\nu} c^{k}(x) \bar{d}^{m}(x) \gamma_{\mu} C \bar{c}^{T n}(x)\right],
\end{aligned}
$$

$\sigma_{\mu \nu}^{t}=\frac{i}{2}\left[\gamma_{\mu}^{t}, \gamma_{\nu}^{t}\right], \quad \sigma_{\mu \nu}^{v}=\frac{i}{2}\left[\gamma_{\mu}^{v}, \gamma_{\nu}^{t}\right], \quad \gamma_{\mu}^{v}=\gamma \cdot t t_{\mu}, \quad \gamma_{\mu}^{t}=\gamma_{\mu}-$ $\gamma \cdot t t_{\mu}, t^{\mu}=(1, \overrightarrow{0})$, the $i, j, k, m, n$ are color indexes, the $C$ is the charge conjugation matrix, the subscripts \pm denote the positive charge conjugation and negative charge conjugation, respectively, the superscripts or subscripts $P, S$, $A(\tilde{A})$ and $V(\tilde{V})$ denote the pseudoscalar, scalar, axialvector and vector diquark, and antidiquark operators, respectively $[17,20,26,27,29,31]$. For the currents, $J_{S S}(x), J_{\tilde{A} \tilde{A}}(x)$, $J_{\tilde{V} \tilde{V}}(x), J_{-, \mu}^{S A}(x), J_{-, \mu \nu}^{A A}(x), J_{-, \mu}^{\tilde{A A}}(x), J_{-, \mu}^{\tilde{V} V}(x), J_{+, \mu}^{S A}(x)$, and $J_{+, \mu \nu}^{A A}(x)$, we update the old calculations. For the currents, $J_{A A}(x), J_{V V}(x), J_{P P}(x), J_{-, \mu \nu}^{S \tilde{A}}(x), J_{-, \mu \nu}^{V V}(x), J_{-, \mu}^{P V}(x), J_{+, \mu \nu}^{S \tilde{A}}(x)$, $J_{+, \mu}^{\tilde{V} V}(x), J_{+, \mu}^{\tilde{A A}}(x), J_{+, \mu}^{P V}(x)$, and $J_{+, \mu \nu}^{V V}(x)$, we obtain new results. Under the parity transform $\hat{P}$, the current operators have the properties,

$$
\begin{gathered}
\hat{P} J(x) \hat{P}^{-1}=+J(\tilde{x}), \\
\hat{P} J_{\mu}(x) \hat{P}^{-1}=-J^{\mu}(\tilde{x}), \\
\hat{P} J_{\mu \nu}^{S \tilde{A}}(x) \hat{P}^{-1}=-J_{S \tilde{A}}^{\mu \nu}(\tilde{x}), \\
\hat{P} J_{\mu \nu}^{A A / V V}(x) \hat{P}^{-1}=+J_{A A / V V}^{\mu \nu}(\tilde{x}),
\end{gathered}
$$

where $x^{\mu}=(t, \vec{x})$ and $\tilde{x}^{\mu}=(t,-\vec{x})$, and we have neglected the other superscripts and subscripts of the current operators.

The current operators $J(x), J_{\mu}(x)$, and $J_{\mu \nu}(x)$ have the symbolic quark structure $\bar{c} c \bar{d} u$ with the isospin $I=1$ and
$I_{3}=1$. Other currents in the isospin multiplets can be constructed analogously; for example, we can write down the corresponding isospin singlet current for the $J_{S S}(x)$ directly,

$$
\begin{aligned}
J_{S S}^{I=0}(x)= & \frac{\varepsilon^{i j k} \varepsilon^{i m n}}{\sqrt{2}}\left[u^{T j}(x) C \gamma_{5} c^{k}(x) \bar{u}^{m}(x) \gamma_{5} C \bar{c}^{T n}(x)\right. \\
& \left.+d^{T j}(x) C \gamma_{5} c^{k}(x) \bar{d}^{m}(x) \gamma_{5} C \bar{c}^{T n}(x)\right] .
\end{aligned}
$$

In the isospin limit, the current operators with the symbolic quark structures $\bar{c} c \bar{d} u, \bar{c} c \bar{u} d, \bar{c} c \frac{\bar{u} u-\bar{d} d}{\sqrt{2}}, \bar{c} c \frac{\bar{u} u+\bar{d} d}{\sqrt{2}}$ potentially couple to the hidden-charm tetraquark states with degenerated masses; the current operators with $I=1$ and 0 lead to the same QCD sum rules. Thereafter, we will denote the $Z_{c}$ states as the isospin triplet, and the $X$ states as the isospin singlet,

$$
\begin{aligned}
& Z_{c}: \bar{c} c \bar{d} u, \bar{c} c \bar{u} d, \bar{c} c \frac{\bar{u} u-\bar{d} d}{\sqrt{2}}, \\
& X: \bar{c} c \frac{\bar{u} u+\bar{d} d}{\sqrt{2}} .
\end{aligned}
$$

The current operators with the symbolic quark structures $\bar{c} c \frac{\bar{u} u-\bar{d} d}{\sqrt{2}}$ and $\bar{c} c \frac{\bar{u} u+\bar{d} d}{\sqrt{2}}$ have definite charge conjugation. In this article, we will assume that the $\bar{c} c \bar{d} u$ type tetraquark states have the same charge conjugation as their neutral charge partners. 
Under the charge conjugation transform $\hat{C}$, the currents $J(x), J_{\mu}(x)$, and $J_{\mu \nu}(x)$ have the properties,

$$
\begin{aligned}
\hat{C} J(x) \hat{C}^{-1} & =+\left.J(x)\right|_{u \leftrightarrow d}, \\
\hat{C} J_{ \pm, \mu}(x) \hat{C}^{-1} & = \pm\left. J_{ \pm, \mu}(x)\right|_{u \leftrightarrow d}, \\
\hat{C} J_{ \pm, \mu \nu}(x) \hat{C}^{-1} & = \pm\left. J_{ \pm, \mu \nu}(x)\right|_{u \leftrightarrow d},
\end{aligned}
$$

where we have neglected other superscripts and subscripts of the current operators.

At the hadron side, we insert a complete set of intermediate hadronic states with the same quantum numbers as the current operators $J(x), J_{\mu}(x)$, and $J_{\mu \nu}(x)$ into the correlation functions $\Pi(p), \Pi_{\mu \nu}(p)$, and $\Pi_{\mu \nu \alpha \beta}(p)$ to obtain the hadronic representation $[13,14]$ and isolate the ground state hidden-charm tetraquark contributions,

$$
\begin{aligned}
\Pi(p)= & \frac{\lambda_{Z^{+}}^{2}}{M_{Z^{+}}^{2}-p^{2}}+\cdots \\
= & \Pi_{+}\left(p^{2}\right) \\
\Pi_{\mu \nu}(p)= & \frac{\lambda_{Z^{+}}^{2}}{M_{Z^{+}}^{2}-p^{2}}\left(-g_{\mu \nu}+\frac{p_{\mu} p_{\nu}}{p^{2}}\right)+\cdots \\
= & \Pi_{+}\left(p^{2}\right)\left(-g_{\mu \nu}+\frac{p_{\mu} p_{\nu}}{p^{2}}\right)+\cdots \\
\Pi_{\mu \nu \alpha \beta}^{A A,-}(p)= & \frac{\lambda_{Z^{+}}^{2}}{M_{Z^{+}}^{2}\left(M_{Z^{+}}^{2}-p^{2}\right)}\left(p^{2} g_{\mu \alpha} g_{\nu \beta}-p^{2} g_{\mu \beta} g_{\nu \alpha}-g_{\mu \alpha} p_{\nu} p_{\beta}-g_{\nu \beta} p_{\mu} p_{\alpha}+g_{\mu \beta} p_{\nu} p_{\alpha}+g_{\nu \alpha} p_{\mu} p_{\beta}\right) \\
& +\frac{\lambda_{Z^{-}}^{2}}{M_{Z^{-}}^{2}\left(M_{Z^{-}}^{2}-p^{2}\right)}\left(-g_{\mu \alpha} p_{\nu} p_{\beta}-g_{\nu \beta} p_{\mu} p_{\alpha}+g_{\mu \beta} p_{\nu} p_{\alpha}+g_{\nu \alpha} p_{\mu} p_{\beta}\right)+\cdots \\
= & \tilde{\Pi}_{+}\left(p^{2}\right)\left(p^{2} g_{\mu \alpha} g_{\nu \beta}-p^{2} g_{\mu \beta} g_{\nu \alpha}-g_{\mu \alpha} p_{\nu} p_{\beta}-g_{\nu \beta} p_{\mu} p_{\alpha}+g_{\mu \beta} p_{\nu} p_{\alpha}+g_{\nu \alpha} p_{\mu} p_{\beta}\right) \\
& +\tilde{\Pi}_{-}\left(p^{2}\right)\left(-g_{\mu \alpha} p_{\nu} p_{\beta}-g_{\nu \beta} p_{\mu} p_{\alpha}+g_{\mu \beta} p_{\nu} p_{\alpha}+g_{\nu \alpha} p_{\mu} p_{\beta}\right) \\
\Pi_{\mu \nu \alpha \beta}^{S \tilde{A}, \pm}(p)= & \frac{\lambda_{Z^{-}}^{2}}{M_{Z^{-}}^{2}\left(M_{Z^{-}}^{2}-p^{2}\right)}\left(p^{2} g_{\mu \alpha} g_{\nu \beta}-p^{2} g_{\mu \beta} g_{\nu \alpha}-g_{\mu \alpha} p_{\nu} p_{\beta}-g_{\nu \beta} p_{\mu} p_{\alpha}+g_{\mu \beta} p_{\nu} p_{\alpha}+g_{\nu \alpha} p_{\mu} p_{\beta}\right) \\
& +\frac{\lambda_{Z^{+}}^{2}}{M_{Z^{+}}^{2}\left(M_{Z^{+}}^{2}-p^{2}\right)}\left(-g_{\mu \alpha} p_{\nu} p_{\beta}-g_{\nu \beta} p_{\mu} p_{\alpha}+g_{\mu \beta} p_{\nu} p_{\alpha}+g_{\nu \alpha} p_{\mu} p_{\beta}\right)+\cdots \\
= & \tilde{\Pi}_{-}\left(p^{2}\right)\left(p^{2} g_{\mu \alpha} g_{\nu \beta}-p^{2} g_{\mu \beta} g_{\nu \alpha}-g_{\mu \alpha} p_{\nu} p_{\beta}-g_{\nu \beta} p_{\mu} p_{\alpha}+g_{\mu \beta} p_{\nu} p_{\alpha}+g_{\nu \alpha} p_{\mu} p_{\beta}\right) \\
& +\tilde{\Pi}_{+}\left(p^{2}\right)\left(-g_{\mu \alpha} p_{\nu} p_{\beta}-g_{\nu \beta} p_{\mu} p_{\alpha}+g_{\mu \beta} p_{\nu} p_{\alpha}+g_{\nu \alpha} p_{\mu} p_{\beta}\right), \\
\Pi_{\mu \nu \alpha \beta}^{A A,+}(p)= & \frac{\lambda_{Z^{+}}^{2}}{M_{Z^{+}}^{2}-p^{2}}\left(\frac{\tilde{g}_{\mu \alpha} \tilde{g}_{\nu \beta}+\tilde{g}_{\mu \beta} \tilde{g}_{\nu \alpha}}{2}-\frac{\tilde{g}_{\mu \nu} \tilde{g}_{\alpha \beta}}{3}\right)+\cdots, \\
= & \Pi_{+}\left(p^{2}\right)\left(\frac{\tilde{g}_{\mu \alpha} \tilde{g}_{\nu \beta}+\tilde{g}_{\mu \beta} \tilde{g}_{\nu \alpha}-\tilde{g}_{\mu \nu} \tilde{g}_{\alpha \beta}}{3}\right)+\cdots, \\
2 &
\end{aligned}
$$

where $\tilde{g}_{\mu \nu}=g_{\mu \nu}-\frac{p_{\mu} p_{\nu}}{p^{2}}$. We add the superscripts \pm in the correlation functions $\Pi_{\mu \nu \alpha \beta}^{A A,-}(p), \Pi_{\mu \nu \alpha \beta}^{S \tilde{A}, \pm}(p)$, and $\Pi_{\mu \nu \alpha \beta}^{A A,+}(p)$ to denote the positive and negative charge conjugation, respectively, and add the superscripts (subscripts) \pm in the hidden-charm tetraquark states $Z_{c}^{ \pm}$(the $\Pi_{ \pm}\left(p^{2}\right)$ and $\tilde{\Pi}_{ \pm}\left(p^{2}\right)$ components of the correlation functions) to denote the positive and negative parity contributions, respectively. The correlation functions $\Pi_{\mu \nu \alpha \beta}^{V V, \pm}(p)$ and $\Pi_{\mu \nu \alpha \beta}^{A A, \pm}(p)$ have the same tensor structures; we neglect the explicit expressions of the $\Pi_{\mu \nu \alpha \beta}^{V V, \pm}(p)$ for simplicity. The pole residues or current-tetraquark coupling constants $\lambda_{Z^{ \pm}}$are defined by 
TABLE II. The quark structures and corresponding current operators for the hidden-charm tetraquark states.

\begin{tabular}{llc}
\hline \hline$Z_{c}$ & $J^{P C}$ & Currents \\
\hline$[u c]_{S}[\overline{d c}]_{S}$ & $0^{++}$ & $J_{S S}(x)$ \\
{$[u c]_{A}[\overline{d c}]_{A}$} & $0^{++}$ & $J_{A A}(x)$ \\
{$[u c]_{\tilde{A}}[\overline{d c}]_{\tilde{A}}$} & $0^{++}$ & $J_{\tilde{A} \tilde{A}}(x)$ \\
{$[u c]_{V}[\overline{d c}]_{V}$} & $0^{++}$ & $J_{V V}(x)$ \\
{$[u c]_{\tilde{V}}[\overline{d c}]_{\tilde{V}}$} & $0^{++}$ & $J_{\tilde{V} \tilde{V}}(x)$ \\
{$[u c]_{P}[\overline{d c}]_{P}$} & $0^{++}$ & $J_{P P}(x)$ \\
{$[u c]_{S}[\overline{d c}]_{A}-[u c]_{A}[\overline{d c}]_{S}$} & $1^{+-}$ & $J_{-, \mu}^{S A}(x)$ \\
{$[u c]_{A}[\overline{d c}]_{A}$} & $1^{+-}$ & $J_{-, \mu \nu}^{A A}(x)$ \\
{$[u c]_{S}[\overline{d c}]_{\tilde{A}}-[u c]_{\tilde{A}}[\overline{d c}]_{S}$} & $1^{+-}$ & $J_{-, \mu \nu}^{S \tilde{A}}(x)$ \\
{$[u c]_{\tilde{A}}[\overline{d c}]_{A}-[u c]_{A}[\overline{d c}]_{\tilde{A}}$} & $1^{+-}$ & $J_{-, \mu}^{\tilde{A} A}(x)$ \\
{$[u c]_{\tilde{V}}[\overline{d c}]_{V}+[u c]_{V}[\overline{d c}]_{\tilde{V}}$} & $1^{+-}$ & $J_{-, \mu}^{\tilde{V} V}(x)$ \\
{$[u c]_{V}[\overline{d c}]_{V}$} & $1^{+-}$ & $J_{-, \mu \nu}^{V V}(x)$ \\
{$[u c]_{P}[\overline{d c}]_{V}+[u c]_{V}[\overline{d c}]_{P}$} & $1^{+-}$ & $J_{-, \mu}^{P V}(x)$ \\
{$[u c]_{S}[\overline{d c}]_{A}+[u c]_{A}[\overline{d c}]_{S}$} & $1^{++}$ & $J_{+, \mu}^{S A}(x)$ \\
{$[u c]_{S}[\overline{d c}]_{\tilde{A}}+[u c]_{\tilde{A}}[\overline{d c}]_{S}$} & $1^{++}$ & $J_{+, \mu \nu}^{S \tilde{A}}(x)$ \\
{$[u c]_{\tilde{V}}[\overline{d c}]_{V}-[u c]_{V}[\overline{d c}]_{\tilde{V}}$} & $1^{++}$ & $J_{+, \mu}^{\tilde{V} V}(x)$ \\
{$[u c]_{\tilde{A}}[\overline{d c}]_{A}+[u c]_{A}[\overline{d c}]_{\tilde{A}}$} & $1^{++}$ & $J_{+, \mu}^{\tilde{A} A}(x)$ \\
{$[u c]_{P}[\overline{d c}]_{V}-[u c]_{V}[\overline{d c}]_{P}$} & $1^{++}$ & $J_{+, \mu}^{P V}(x)$ \\
{$[u c]_{A}[\overline{d c}]_{A}$} & $2^{++}$ & $J_{+, \mu \nu}^{A A}(x)$ \\
{$[u c]_{V}[\overline{d c}]_{V}$} & $2^{++}$ & $J_{+, \mu \nu}^{V V}(x)$ \\
\hline \hline
\end{tabular}

$$
\begin{aligned}
\left\langle 0|J(0)| Z_{c}^{+}(p)\right\rangle & =\lambda_{Z^{+}}, \\
\left\langle 0\left|J_{\mu}(0)\right| Z_{c}^{+}(p)\right\rangle & =\lambda_{Z^{+}} \varepsilon_{\mu}, \\
\left\langle 0\left|J_{ \pm, \mu \nu}^{S \tilde{A}}(0)\right| Z_{c}^{-}(p)\right\rangle & =\frac{\lambda_{Z^{-}}}{M_{Z^{-}}} \varepsilon_{\mu \nu \alpha \beta} \varepsilon^{\alpha} p^{\beta}, \\
\left\langle 0\left|J_{ \pm, \mu \nu}^{S \tilde{A}}(0)\right| Z_{c}^{+}(p)\right\rangle & =\frac{\lambda_{Z^{+}}}{M_{Z^{+}}}\left(\varepsilon_{\mu} p_{\nu}-\varepsilon_{\nu} p_{\mu}\right), \\
\left\langle 0\left|J_{-, \mu \nu}^{A A / V V}(0)\right| Z_{c}^{+}(p)\right\rangle & =\frac{\lambda_{Z^{+}}}{M_{Z^{+}}} \varepsilon_{\mu \nu \alpha \beta} \varepsilon^{\alpha} p^{\beta}, \\
\left\langle 0\left|J_{-, \mu \nu}^{A A / V V}(0)\right| Z_{c}^{-}(p)\right\rangle & =\frac{\lambda_{Z^{-}}}{M_{Z^{-}}}\left(\varepsilon_{\mu} p_{\nu}-\varepsilon_{\nu} p_{\mu}\right), \\
\left\langle 0\left|J_{+, \mu \nu}^{A A / V V}(0)\right| Z_{c}^{+}(p)\right\rangle & =\lambda_{Z^{+}} \varepsilon_{\mu \nu},
\end{aligned}
$$

where the $\varepsilon_{\mu / \alpha}$ and $\varepsilon_{\mu \nu}$ are the polarization vectors. In this article, we choose the components $\Pi_{+}\left(p^{2}\right)$ and $p^{2} \tilde{\Pi}_{+}\left(p^{2}\right)$ to study the scalar, axialvector, and tensor hidden-charm tetraquark states with the QCD sum rules. In Table II, we present the quark structures and corresponding interpolating currents for the hidden-charm tetraquark states.

Now, we take a short digression to discuss the feasibility of applying the QCD sum rules to study the tetraquark states and $\mathbf{1}_{c} \mathbf{1}_{c}$-type tetraquark states. We usually perform a Fierz rearrangement both in the color space and Diracspinor space to arrange the diquark-antidiquark type currents into a special superposition of the $\mathbf{1}_{c} \mathbf{1}_{c}$-type currents [19]; for example,

$$
\begin{aligned}
J_{+, \mu}^{S A}= & \frac{1}{2 \sqrt{2}}\left\{i \bar{c} i \gamma_{5} c \bar{d} \gamma_{\mu} u-i \bar{c} \gamma_{\mu} c \bar{d} i \gamma_{5} u+\bar{c} u \bar{d} \gamma_{\mu} \gamma_{5} c\right. \\
& -\bar{c} \gamma_{\mu} \gamma_{5} u \bar{d} c-i \bar{c} \gamma^{\nu} \gamma_{5} c \bar{d} \sigma_{\mu \nu} u+i \bar{c} \sigma_{\mu \nu} c \bar{d} \gamma^{\nu} \gamma_{5} u \\
& \left.-i \bar{c} \sigma_{\mu \nu} \gamma_{5} u \bar{d} \gamma^{\nu} c+i \bar{c} \gamma^{\nu} u \bar{d} \sigma_{\mu \nu} \gamma_{5} c\right\} .
\end{aligned}
$$

In fact, the barrier or spatial separation between the diquark and antidiquark pair frustrates the Fierz rearrangements [32-35]. If we neglect those frustrations, the diquarkantidiquark type currents and $\mathbf{1}_{c} \mathbf{1}_{c}$-type currents can be rearranged into each other freely; they are all four-quark currents.

In the correlation functions for the $\mathbf{1}_{c} \mathbf{1}_{c}$-type currents, Lucha, et al. assert that the Feynman diagrams can be divided into or separated into factorizable and nonfactorizable diagrams in the color space in the operator product expansion. The contributions at the order $\mathcal{O}\left(\alpha_{s}^{k}\right)$ with $k \leq 1$, which are factorizable in the color space, are exactly canceled out by the two-meson scattering states at the hadron side. The nonfactorizable diagrams, if they have a Landau singularity, begin to make contributions to the $\left(\mathbf{1}_{c} \mathbf{1}_{c}\right.$-type) tetraquark states and the $\left(\mathbf{1}_{c} \mathbf{1}_{c}\right.$-type) tetraquark states begin to receive contributions at the order $\mathcal{O}\left(\alpha_{s}^{2}\right)[36]$.

In Ref. [37], I refute the assertion of Lucha, et al. in order and in details, and use two examples to illustrate that the two-meson scattering states cannot saturate the QCD sum rules, while the $\mathbf{1}_{c} \mathbf{1}_{c}$-type tetraquark states can saturate the QCD sum rules, the $\mathbf{1}_{c} \mathbf{1}_{c}$-type tetraquark states begin to receive contributions at the order $\mathcal{O}\left(\alpha_{s}^{0} / \alpha_{s}^{1}\right)$ rather than at the order $\mathcal{O}\left(\alpha_{s}^{2}\right)$.

The two-meson scattering state and $\mathbf{1}_{c} \mathbf{1}_{c}$-type tetraquark state both have four valence quarks, which form two colorneutral clusters; we cannot distinguish the contributions based on the two color-neutral clusters in the factorizable Feynman diagrams. It is questionable to assert that the factorizable Feynman diagrams only make contributions to the two-meson scattering states. The Landau equation serves as a kinematical equation in the momentum space and is independent of the factorizable and nonfactorizable properties of the Feynman diagrams in color space [38]. The Landau equation cannot exclude the factorizable Feynman diagrams in color space, as they are nonfactorizable in the momentum space and also have Landau singularities.

The quarks and gluons are confined objects; they cannot be put on the mass shell. It is questionable to apply the Landau equation to study the Feynman diagrams in the QCD sum rules. Furthermore, we carry out the operator product expansion in the deep Euclidean region $P^{2}=-p^{2} \rightarrow \infty$, where the Landau singularities cannot exist. There are other negative outcomes, which involve 
applying the Landau equation to study the $\left(\mathbf{1}_{c} \mathbf{1}_{c}\right.$-type) tetraquark states; for detailed discussions about this subject, one can consult Ref. [37].

In Ref. [39], we study the $Z_{c}(3900)$ with the QCD sum rules in detail by including all the two-particle scattering state contributions according to the Fierz rearrangement in Eq. (13) and observe that the two-particle scattering state contributions cannot saturate the QCD sum rules at the hadron side. The contribution of the $Z_{c}(3900)$ plays an unsubstitutable role; we can saturate the QCD sum rules with or without the two-particle scattering state contributions. We obtain the conclusion that it is feasible to apply the QCD sum rules to study the diquark-antidiquark type tetraquark states, which begin to receive contributions at the order $\mathcal{O}\left(\alpha_{s}^{0}\right)$, not at the order $\mathcal{O}\left(\alpha_{s}^{2}\right)$.

Now let us go back to correlation functions $\Pi(p)$, $\Pi_{\mu \nu}(p)$, and $\Pi_{\mu \nu \alpha \beta}(p)$ defined in Eq. (3). At the QCD side, we carry out the operator product expansion for the correlation functions $\Pi(p), \Pi_{\mu \nu}(p)$, and $\Pi_{\mu \nu \alpha \beta}(p)$ up to the vacuum condensates of dimension 10 consistently, take into account the vacuum condensates $\langle\bar{q} q\rangle,\left\langle\frac{\alpha_{s} G G}{\pi}\right\rangle,\left\langle\bar{q} g_{s} \sigma G q\right\rangle$, $\langle\bar{q} q\rangle^{2}, g_{s}^{2}\langle\bar{q} q\rangle^{2},\langle\bar{q} q\rangle\left\langle\frac{\alpha_{s} G G}{\pi}\right\rangle,\langle\bar{q} q\rangle\left\langle\bar{q} g_{s} \sigma G q\right\rangle,\left\langle\bar{q} g_{s} \sigma G q\right\rangle^{2}$, and $\langle\bar{q} q\rangle^{2}\left\langle\frac{\alpha_{s} G G}{\pi}\right\rangle$, and obtain the QCD spectral densities $\rho(s)$ through the dispersion relation. The contributions of the terms $g_{s}^{2}\langle\bar{q} q\rangle^{2}$ are tiny and neglected in most of the QCD sum rules, as they are not associated with the Borel parameters of the forms $\frac{1}{T^{2}}, \frac{1}{T^{4}}, \frac{1}{T^{6}}, \cdots$, which amplify themselves at small values of the $T^{2}$ and play an important role in determining the Borel windows. There are terms of the forms $\left\langle\bar{q}_{j} \sigma_{\mu \nu} q_{i}\right\rangle$ and $\left\langle\bar{q}_{j} \gamma_{\mu} q_{i}\right\rangle$ in the full light quark propagators [17], which absorb the gluons emitted from the other quark lines to form $\left\langle\bar{q}_{j} g_{s} G_{\alpha \beta}^{a} t_{m n}^{a} \sigma_{\mu \nu} q_{i}\right\rangle$ and $\left\langle\bar{q}_{j} \gamma_{\mu} q_{i} g_{s} D_{\nu} G_{\alpha \beta}^{a} t_{m n}^{a}\right\rangle$ to make contributions to the mixed condensate and four-quark condensate $\left\langle\bar{q} g_{s} \sigma G q\right\rangle$ and $g_{s}^{2}\langle\bar{q} q\rangle^{2}$, respectively. The four-quark condensate $g_{s}^{2}\langle\bar{q} q\rangle^{2}$ comes from the terms $\left\langle\bar{q} \gamma_{\mu} t^{a} q g_{s} D_{\eta} G_{\lambda \tau}^{a}\right\rangle,\left\langle\bar{q}_{j} D_{\mu}^{\dagger} D_{\nu}^{\dagger} D_{\alpha}^{\dagger} q_{i}\right\rangle$, and $\left\langle\bar{q}_{j} D_{\mu} D_{\nu} D_{\alpha} q_{i}\right\rangle$ rather than from the perturbative $\mathcal{O}\left(\alpha_{s}\right)$ corrections for the four-quark condensate $\langle\bar{q} q\rangle^{2}$, where $D_{\alpha}=\partial_{\alpha}-i g_{s} G_{\alpha}^{n} t^{n}, t^{n}=\frac{\lambda^{n}}{2}$, and the $\lambda^{n}$ is the Gell-Mann matrix. The strong coupling constant $\alpha_{s}(\mu)=\frac{g_{s}^{2}(\mu)}{4 \pi}$ appears at the tree level, which is energy scale dependent. One can consult Ref. [17] for the technical details. Furthermore, we recalculate the higher dimensional vacuum condensates using the formula $t_{i j}^{a} t_{m n}^{a}=-\frac{1}{6} \delta_{i j} \delta_{m n}+\frac{1}{2} \delta_{j m} \delta_{\text {in }}$ and obtain slightly different expressions compared to the old calculations.

As we have obtained both the hadron spectral representations and the QCD spectral representations, now we match the hadron side with the QCD side of the components $\Pi_{+}\left(p^{2}\right)$ and $p^{2} \tilde{\Pi}_{+}\left(p^{2}\right)$ of the correlation functions $\Pi(p), \Pi_{\mu \nu}(p)$ and $\Pi_{\mu \nu \alpha \beta}(p)$ below the continuum thresholds $s_{0}$ and perform the Borel transform with respect to $P^{2}=-p^{2}$ to obtain the QCD sum rules,

$$
\lambda_{Z^{+}}^{2} \exp \left(-\frac{M_{Z^{+}}^{2}}{T^{2}}\right)=\int_{4 m_{c}^{2}}^{s_{0}} d s \rho(s) \exp \left(-\frac{s}{T^{2}}\right)
$$

The explicit expressions of the QCD spectral densities $\rho(s)$ are available upon request by contacting me via email.

We derive Eq. (14) with respect to $\tau=\frac{1}{T^{2}}$ and obtain the QCD sum rules for the masses of the scalar, axialvector, and tensor hidden-charm tetraquark states $Z_{c}$ or $X$,

$$
M_{Z^{+}}^{2}=-\frac{\int_{4 m_{c}^{2}}^{s_{0}} d s \frac{d}{d \tau} \rho(s) \exp (-\tau s)}{\int_{4 m_{c}^{2}}^{s_{0}} d s \rho(s) \exp (-\tau s)} .
$$

\section{NUMERICAL RESULTS AND DISCUSSIONS}

In this article, we neglect the small $u$ and $d$ quark masses. The heavy quark ( $\overline{\mathrm{MS}})$ mass $m_{c}(\mu)$ and the vacuum condensates depend on the energy scale $\mu$, so the QCD spectral densities $\rho(s, \mu)$ depend on the energy scale $\mu$. The thresholds $4 m_{c}^{2}(\mu)$ also depend on the energy scale $\mu$, and we cannot extract the masses of the hidden-charm tetraquark states from the energy scale independent QCD sum rules and have to choose the best energy scales to extract the tetraquark masses. The energy-scale dependence of the input parameters at the QCD side can be written as

$$
\begin{aligned}
\langle\bar{q} q\rangle(\mu) & =\langle\bar{q} q\rangle(1 \mathrm{GeV})\left[\frac{\alpha_{s}(1 \mathrm{GeV})}{\alpha_{s}(\mu)}\right]^{\frac{12}{33-2 n_{f}}}, \\
\left\langle\bar{q} g_{s} \sigma G q\right\rangle(\mu) & =\left\langle\bar{q} g_{s} \sigma G q\right\rangle(1 \mathrm{GeV})\left[\frac{\alpha_{s}(1 \mathrm{GeV})}{\alpha_{s}(\mu)}\right]^{\frac{2}{33-2 n_{f}}}, \\
m_{c}(\mu)= & m_{c}\left(m_{c}\right)\left[\frac{\alpha_{s}(\mu)}{\alpha_{s}\left(m_{c}\right)}\right]^{\frac{12}{33-2 n_{f}}}, \\
\alpha_{s}(\mu)= & \frac{1}{b_{0} t}\left[1-\frac{b_{1}}{b_{0}^{2}} \frac{\log t}{t}\right. \\
& \left.+\frac{b_{1}^{2}\left(\log ^{2} t-\log t-1\right)+b_{0} b_{2}}{b_{0}^{4} t^{2}}\right],
\end{aligned}
$$

from the renormalization group equation, where $t=$ $\log \frac{\mu^{2}}{\Lambda_{\mathrm{QCD}}^{2}}, \quad b_{0}=\frac{33-2 n_{f}}{12 \pi}, \quad b_{1}=\frac{153-19 n_{f}}{24 \pi^{2}}, \quad b_{2}=\frac{2857-\frac{5033}{9} n_{f}+\frac{325}{27} n_{f}^{2}}{128 \pi^{3}}$, $\Lambda_{\mathrm{QCD}}=210 \mathrm{MeV}, 292 \mathrm{MeV}$, and $332 \mathrm{MeV}$ for the flavors $n_{f}=5,4$, and 3 , respectively $[2,40]$. In the present work, as the $c$ quark is involved, we take the flavor $n_{f}=4$.

At the initial points, we take the standard values of the vacuum condensates $\langle\bar{q} q\rangle=-(0.24 \pm 0.01 \mathrm{GeV})^{3}$, $\left\langle\bar{q} g_{s} \sigma G q\right\rangle=m_{0}^{2}\langle\bar{q} q\rangle, \quad m_{0}^{2}=(0.8 \pm 0.1) \mathrm{GeV}^{2}, \quad\left\langle\frac{\alpha_{s} G G}{\pi}\right\rangle=$ $(0.33 \mathrm{GeV})^{4}$ at the energy scale $\mu=1 \mathrm{GeV}$ [13-15] and take the $\overline{\mathrm{MS}}$ mass $m_{c}\left(m_{c}\right)=(1.275 \pm 0.025) \mathrm{GeV}$ from the Particle Data Group [2].

The hidden-bottom or hidden-charm tetraquark states can be described by a double-well potential model; the heavy quark $Q$ (heavy antiquark $\bar{Q}$ ) serves as a static well 
potential and attracts the light quark $q$ (light antiquark $\bar{q}$ ) to form a diquark (antidiquark) in the color antitriplet (triplet) channel. The diquark-antidiquark type tetraquark states, which are excellent candidates for the $X, Y, Z$ states, are characterized by the effective heavy quark mass $\mathbb{M}_{Q}$ and the virtuality $V=\sqrt{M_{X / Y / Z}^{2}-\left(2 \mathbb{M}_{Q}\right)^{2}}$. If we choose the energy scale $[19,20]$,

$$
\mu^{2}=V^{2}=\mathcal{O}\left(T^{2}\right),
$$

then we obtain the formula $\mu=\sqrt{M_{X / Y / Z}^{2}-\left(2 M_{Q}\right)^{2}}$ to determine the best energy scales for the QCD sum rules. At the ideal energy scales, we can enhance the pole contributions at the hadron side remarkably and improve the convergent behaviors of the operator product expansion at the QCD side remarkably. In the present work, we use the energy scale formula $\mu=\sqrt{M_{X / Y / Z}^{2}-\left(2 \mathbb{M}_{c}\right)^{2}}$ with the updated effective $c$-quark mass $\mathbb{M}_{c}=1.82 \mathrm{GeV}$ to determine the ideal energy scales for the QCD sum rules [41].

At the QCD side, we carry out the operator product expansion at the large spacelike region,

$$
x_{0} \sim|\vec{x}| \sim \frac{1}{\sqrt{P^{2}}} \ll \frac{1}{\Lambda_{\mathrm{QCD}}} ;
$$

the anomalous dimensions $\gamma_{J}$ of the interpolating currents $J(x), J_{\mu}(x)$, and $J_{\mu \nu}(x)$ lead to a factor multiplying the correlation functions at the QCD side [42]. After the Borel transformation, the factor is changed to

$$
\left[\frac{\alpha_{s}\left(P^{2}\right)}{\alpha_{s}\left(\mu^{2}\right)}\right]^{2 \gamma_{J}} \rightarrow\left[\frac{\alpha_{s}\left(T^{2}\right)}{\alpha_{s}\left(\mu^{2}\right)}\right]^{2 \gamma_{J}}
$$

If we choose $\mu^{2}=\mathcal{O}\left(T^{2}\right)$, the factor is neglected. In fact, the present approach weakens the energy scale dependence of the QCD sum rules, although we cannot obtain energy scale independent QCD sum rules.

The continuum threshold parameters are not completely free parameters and cannot be determined by the QCD sum rules themselves completely. We often consult the experimental data in choosing the continuum threshold parameters. The $Z_{c}(4430)$ can be assigned to be the first radial excitation of the $Z_{c}(3900)$ according to the analogous decays,

$$
\begin{aligned}
& Z_{c}^{ \pm}(3900) \rightarrow J / \psi \pi^{ \pm}, \\
& Z_{c}^{ \pm}(4430) \rightarrow \psi^{\prime} \pi^{ \pm},
\end{aligned}
$$

and the analogous mass gaps $M_{Z_{c}(4430)}-M_{Z_{c}(3900)}=$ $591 \mathrm{MeV}$ and $M_{\psi^{\prime}}-M_{J / \psi}=589 \mathrm{MeV}$ from the Particle Data Group [2,43-45]. Thereafter, we will use the superscripts \pm to denote the electric charges. The energy gap
$M_{X(4500)}-M_{X(3915)}=588 \mathrm{MeV}$ from the Particle Data Group [2]; the $X(3915)$ and $X(4500)$ can be assigned as the ground state and the first radial excited state of the axialvector-diquark-axialvector-antidiquark type scalar $\operatorname{cs} \bar{c} \bar{s}$ tetraquark states [28]. The QCD sum rules also support such assignments [26]. Recently, the LHCb Collaboration performed an angular analysis of the $B^{0} \rightarrow$ $J / \psi K^{+} \pi^{-}$decays and observed two possible structures near $m\left(J / \psi \pi^{-}\right)=4200 \mathrm{MeV}$ and $4600 \mathrm{MeV}$, respectively [46]. There have been two tentative assignments for the structure $Z_{c}(4600)$, the vector tetraquark state with $J^{P C}=$ $1^{--}[47]$ and the first radial excited axialvector tetraquark state with $J^{P C}=1^{+-}[30,31]$. If the dominant Fock component of the $Z_{c}(4600)$ is an axialvector tetraquark state, the energy gap between the ground state $Z_{c}(4020)$ and the first radial excited state $Z_{c}(4600)$ is about $M_{Z_{c}(4600)}-M_{Z_{c}(4020)}=576 \mathrm{MeV}$ from the Particle Data Group [2].

In the present work, we tentatively choose the continuum threshold parameters as $\sqrt{s_{0}}=M_{Z}+0.58 / 0.59 \mathrm{GeV}$ and vary the continuum threshold parameters $s_{0}$ and Borel parameters $T^{2}$ to satisfy the following four criteria:

(i) Pole dominance at the hadron side;

(ii) Convergence of the operator product expansion;

(iii) Appearance of the Borel platforms;

(iv) Satisfying the energy scale formula, via trial and error.

The pole dominance at the hadron side and convergence of the operator product expansion at the QCD side are two basic criteria for the QCD sum rules; we should satisfy the two basic criteria to obtain reliable QCD sum rules. Furthermore, we should obtain Borel platforms to avoid additional uncertainties originate from the Borel parameters. The pole contributions (PC) or ground state tetraquark contributions are defined by

$$
\mathrm{PC}=\frac{\int_{4 m_{c}^{2}}^{s_{0}} d s \rho(s) \exp \left(-\frac{s}{T^{2}}\right)}{\int_{4 m_{c}^{2}}^{\infty} d s \rho(s) \exp \left(-\frac{s}{T^{2}}\right)},
$$

while the contributions of the vacuum condensates $D(n)$ of dimension $n$ are defined by

$$
D(n)=\frac{\int_{4 m_{c}^{2}}^{s_{0}} d s \rho_{n}(s) \exp \left(-\frac{s}{T^{2}}\right)}{\int_{4 m_{c}^{2}}^{s_{0}} d s \rho(s) \exp \left(-\frac{s}{T^{2}}\right)},
$$

as we only study the ground state contributions. At the QCD side of the correlation functions $\Pi(p), \Pi_{\mu \nu}(p)$, and $\Pi_{\mu \nu \alpha \beta}(p)$, there are four full quark propagators, i.e., two heavy quark propagators and two light quark propagators. If each heavy quark line emits a gluon and each light quark line contributes a quark-antiquark pair, we obtain a quarkgluon operator $G G \bar{u} u \bar{d} d$ of dimension 10 , so we should calculate the vacuum condensates at least up to dimension 10 to judge the convergent behavior of the operator product 
TABLE III. The Borel parameters, continuum threshold parameters, energy scales of the QCD spectral densities, pole contributions, and the contributions of the vacuum condensates of dimension 10 for the ground state hiddencharm tetraquark states.

\begin{tabular}{lcccccc}
\hline \hline$Z_{c}\left(X_{c}\right)$ & $J^{P C}$ & $T^{2}\left(\mathrm{GeV}^{2}\right)$ & $\sqrt{s_{0}}(\mathrm{GeV})$ & $\mu(\mathrm{GeV})$ & Pole & $|D(10)|$ \\
\hline$[u c]_{S}[\overline{d c}]_{S}$ & $0^{++}$ & $2.7-3.1$ & $4.40 \pm 0.10$ & 1.3 & $(40-63) \%$ & $<1 \%$ \\
{$[u c]_{A}[\overline{d c}]_{A}$} & $0^{++}$ & $2.8-3.2$ & $4.52 \pm 0.10$ & 1.5 & $(40-63) \%$ & $\leq 1 \%$ \\
{$[u c]_{\tilde{A}}[\overline{d c}]_{\tilde{A}}$} & $0^{++}$ & $3.1-3.5$ & $4.55 \pm 0.10$ & 1.6 & $(42-62) \%$ & $<1 \%$ \\
{$[u c]_{V}[\overline{d c}]_{V}$} & $0^{++}$ & $3.7-4.1$ & $5.22 \pm 0.10$ & 2.9 & $(41-60) \%$ & $\ll 1 \%$ \\
{$[u c]_{\tilde{V}}[\overline{d c}]_{\tilde{V}}$} & $0^{++}$ & $4.9-5.7$ & $5.90 \pm 0.10$ & 3.9 & $(41-61) \%$ & $\ll 1 \%$ \\
{$[u c]_{P}[\overline{d c}]_{P}$} & $0^{++}$ & $5.2-6.0$ & $6.03 \pm 0.10$ & 4.1 & $(40-60) \%$ & $\ll 1 \%$ \\
{$[u c]_{S}[\overline{d c}]_{A}-[u c]_{A}[\overline{d c}]_{S}$} & $1^{+-}$ & $2.7-3.1$ & $4.40 \pm 0.10$ & 1.4 & $(40-63) \%$ & $<1 \%$ \\
{$[u c]_{A}\left[[\overline{d c}]_{A}\right.$} & $1^{+-}$ & $3.3-3.7$ & $4.60 \pm 0.10$ & 1.7 & $(40-59) \%$ & $\ll 1 \%$ \\
{$[u c]_{S}[\overline{d c}]_{\tilde{A}}-[u c]_{\tilde{\tilde{C}}}[\overline{d c}]_{S}$} & $1^{+-}$ & $3.3-3.7$ & $4.60 \pm 0.10$ & 1.7 & $(40-59) \%$ & $\ll 1 \%$ \\
{$[u c]_{\tilde{A}}[\overline{d c}]_{A}-[u c]_{A}[\overline{d c}]_{\tilde{A}}$} & $1^{+-}$ & $3.2-3.6$ & $4.60 \pm 0.10$ & 1.7 & $(41-61) \%$ & $\ll 1 \%$ \\
{$[u c]_{\tilde{V}}[\overline{d c}]_{V}+[u c]_{V}[\overline{d c}]_{\tilde{V}}$} & $1^{+-}$ & $3.7-4.1$ & $5.25 \pm 0.10$ & 2.9 & $(41-60) \%$ & $\ll 1 \%$ \\
{$[u c]_{V}[\overline{d c}]_{V}$} & $1^{+-}$ & $5.1-5.9$ & $6.00 \pm 0.10$ & 4.1 & $(41-60) \%$ & $\ll 1 \%$ \\
{$[u c]_{P}[\overline{d c}]_{V}+[u c]_{V}[\overline{d c}]_{P}$} & $1^{+-}$ & $5.1-5.9$ & $6.00 \pm 0.10$ & 4.1 & $(41-60) \%$ & $\ll 1 \%$ \\
{$[u c]_{S}[\overline{d c}]_{A}+[u c]_{A}[\overline{d c}]_{S}$} & $1^{++}$ & $2.7-3.1$ & $4.40 \pm 0.10$ & 1.4 & $(40-62) \%$ & $\ll 1 \%$ \\
{$[u c]_{S}\left[[\overline{d c}]_{\tilde{A}}+[u c]_{\tilde{A}}[\overline{d c}]_{S}\right.$} & $1^{++}$ & $3.3-3.7$ & $4.60 \pm 0.10$ & 1.7 & $(40-59) \%$ & $\ll 1 \%$ \\
{$[u c]_{\tilde{V}}[\overline{d c}]_{V}-[u c]_{V}[\overline{d c}]_{\tilde{V}}$} & $1^{++}$ & $2.8-3.2$ & $4.62 \pm 0.10$ & 1.8 & $(40-63) \%$ & $<2 \%$ \\
{$[u c]_{\tilde{A}}[\overline{d c}]_{A}+[u c]_{A}[\overline{d c}]_{\tilde{A}}$} & $1^{++}$ & $4.6-5.3$ & $5.73 \pm 0.10$ & 3.7 & $(40-60) \%$ & $\ll 1 \%$ \\
{$[u c]_{P}[\overline{d c}]_{V}-[u c]_{V}[\overline{d c}]_{P}$} & $1^{++}$ & $5.1-5.9$ & $6.00 \pm 0.10$ & 4.1 & $(40-60) \%$ & $\ll 1 \%$ \\
{$[u c]_{A}[\overline{d c}]_{A}$} & $2^{++}$ & $3.3-3.7$ & $4.65 \pm 0.10$ & 1.8 & $(40-60) \%$ & $<1 \%$ \\
{$[u c]_{V}[\overline{d c}]_{V}$} & $2^{++}$ & $5.0-5.8$ & $5.95 \pm 0.10$ & 4.0 & $(40-60) \%$ & $\ll 1 \%$ \\
\hline \hline
\end{tabular}

expansion. The vacuum condensates $\left\langle\bar{q} g_{s} \sigma G q\right\rangle^{2}$ and $\langle\bar{q} q\rangle^{2}\left\langle\frac{\alpha_{s} G G}{\pi}\right\rangle$ are associated with $\frac{1}{T^{2}}, \frac{1}{T^{4}}$, and $\frac{1}{T^{6}}$; they play an important role in determining the Borel windows, although in the Borel windows, they are of minor importance. In the present work, we require the contributions $|D(10)| \sim 1 \%$ at the Borel windows.

Finally, we obtain the Borel windows, continuum threshold parameters, energy scales of the QCD spectral densities, pole contributions, and the contributions of the vacuum condensates of dimension 10 , which are shown explicitly in Table III. From the Table, we can see that the pole contributions are about (40-60)\% at the hadron side; the pole dominance condition is well satisfied. At the QCD side, the contributions of the vacuum condensates of dimension 10 are $|D(10)| \leq 1 \%$ or $\ll 1 \%$ except for $|D(10)|<2 \%$ for the $[u c]_{\tilde{V}}[\overline{d c}]_{V}-[u c]_{V}[\overline{d c}]_{\tilde{V}}$ tetraquark state with the spin-parity-charge-conjugation $J^{P C}=1^{++}$; the convergent behavior of the operator product expansion is very good.

We take into account all the uncertainties of the input parameters and obtain the masses and pole residues of the scalar, axialvector, tensor hidden-charm tetraquark states, which are shown explicitly in Table IV. From Tables III-IV, we can see that the energy scale formula $\mu=\sqrt{M_{X / Y / Z}^{2}-\left(2 \mathbb{M}_{c}\right)^{2}}$ is well satisfied. In Fig. 1, we plot
TABLE IV. The masses and pole residues of the ground state hidden-charm tetraquark states.

\begin{tabular}{lccc}
\hline \hline$Z_{c}\left(X_{c}\right)$ & $J^{P C}$ & $M_{Z}(\mathrm{GeV})$ & $\lambda_{Z}\left(\mathrm{GeV}^{5}\right)$ \\
\hline$[u c]_{S}[\overline{d c}]_{S}$ & $0^{++}$ & $3.88 \pm 0.09$ & $(2.07 \pm 0.35) \times 10^{-2}$ \\
{$[u c]_{A}[\overline{d c}]_{A}$} & $0^{++}$ & $3.95 \pm 0.09$ & $(4.49 \pm 0.77) \times 10^{-2}$ \\
{$[u c]_{\tilde{A}}[\overline{d c}]_{\tilde{A}}$} & $0^{++}$ & $3.98 \pm 0.08$ & $(4.30 \pm 0.63) \times 10^{-2}$ \\
{$[u c]_{V}[\overline{d c}]_{V}$} & $0^{++}$ & $4.65 \pm 0.09$ & $(1.35 \pm 0.22) \times 10^{-1}$ \\
{$[u c]_{\tilde{V}}[\overline{d c}]_{\tilde{V}}$} & $0^{++}$ & $5.35 \pm 0.09$ & $(4.87 \pm 0.51) \times 10^{-1}$ \\
{$[u c]_{P}[\overline{d c}]_{P}$} & $0^{++}$ & $5.49 \pm 0.09$ & $(2.11 \pm 0.21) \times 10^{-1}$ \\
{$[u c]_{S}[\overline{d c}]_{A}-[u c]_{A}[\overline{d c}]_{S}$} & $1^{+-}$ & $3.90 \pm 0.08$ & $(2.09 \pm 0.33) \times 10^{-2}$ \\
{$[u c]_{A}[\overline{d c}]_{A}$} & $1^{+-}$ & $4.02 \pm 0.09$ & $(3.00 \pm 0.45) \times 10^{-2}$ \\
{$[u c]_{S}[\overline{d c}]_{\tilde{A}}-[u c]_{\tilde{A}}[\overline{d c}]_{S}$} & $1^{+-}$ & $4.01 \pm 0.09$ & $(3.02 \pm 0.45) \times 10^{-2}$ \\
{$[u c]_{\tilde{A}}[\overline{d c}]_{A}-[u c]_{A}[\overline{d c}]_{\tilde{A}}$} & $1^{+-}$ & $4.02 \pm 0.09$ & $(6.09 \pm 0.90) \times 10^{-2}$ \\
{$[u c]_{\tilde{V}}[\overline{d c}]_{V}+[u c]_{V}[\overline{d c}]_{\tilde{V}}$} & $1^{+-}$ & $4.66 \pm 0.10$ & $(1.18 \pm 0.21) \times 10^{-1}$ \\
{$[u c]_{V}[\overline{d c}]_{V}$} & $1^{+-}$ & $5.46 \pm 0.09$ & $(1.72 \pm 0.17) \times 10^{-1}$ \\
{$[u c]_{P}[\overline{d c}]_{V}+[u c]_{V}[\overline{d c}]_{P}$} & $1^{+-}$ & $5.45 \pm 0.09$ & $(1.87 \pm 0.19) \times 10^{-1}$ \\
{$[u c]_{S}[\overline{d c}]_{A}+[u c]_{A}[\overline{d c}]_{S}$} & $1^{++}$ & $3.91 \pm 0.08$ & $(2.10 \pm 0.34) \times 10^{-2}$ \\
{$[u c]_{S}[\overline{d c}]_{\tilde{A}}+[u c]_{\tilde{n}}[\overline{d c}]_{S}$} & $1^{++}$ & $4.02 \pm 0.09$ & $(3.01 \pm 0.45) \times 10^{-2}$ \\
{$[u c]_{\tilde{V}}[\overline{d c}]_{V}-[u c]_{V}[\overline{d c}]_{\tilde{V}}$} & $1^{++}$ & $4.08 \pm 0.09$ & $(3.67 \pm 0.67) \times 10^{-2}$ \\
{$[u c]_{\tilde{A}}[\overline{d c}]_{A}+[u c]_{A}[\overline{d c}]_{\tilde{A}}$} & $1^{++}$ & $5.19 \pm 0.09$ & $(2.12 \pm 0.24) \times 10^{-1}$ \\
{$[u c]_{P}[\overline{d c}]_{V}-[u c]_{V}[\overline{d c}]_{P}$} & $1^{++}$ & $5.46 \pm 0.09$ & $(1.89 \pm 0.19) \times 10^{-1}$ \\
{$[u c]_{A}[\overline{d c}]_{A}$} & $2^{++}$ & $4.08 \pm 0.09$ & $(4.67 \pm 0.68) \times 10^{-2}$ \\
{$[u c]_{V}[\overline{d c}]_{V}$} & $2^{++}$ & $5.40 \pm 0.09$ & $(2.32 \pm 0.25) \times 10^{-1}$ \\
\hline \hline & & & \\
& & &
\end{tabular}



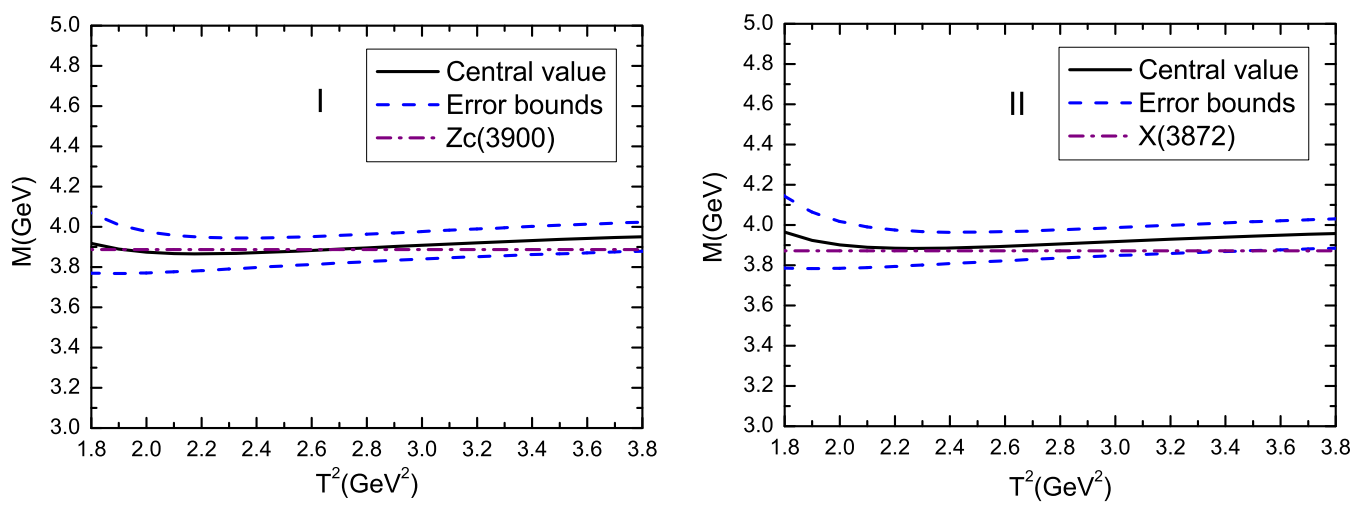

FIG. 1. The masses of the $[u c]_{S}[\overline{d c}]_{A}-[u c]_{A}[\overline{d c}]_{S}(\mathrm{I})$ and $[u c]_{S}[\overline{d c}]_{A}+[u c]_{A}[\overline{d c}]_{S}(\mathrm{II})$ axialvector tetraquark states with variations of the Borel parameters $T^{2}$.

the masses of the $[u c]_{S}[\overline{d c}]_{A}-[u c]_{A}[\overline{d c}]_{S}$ and $[u c]_{S}[\overline{d c}]_{A}+$ $[u c]_{A}[\overline{d c}]_{S}$ axialvector tetraquark states with variations of the Borel parameters at much larger ranges than the Borel widows as an example. From the figure, we can see that there appear platforms in the Borel windows indeed. Now the four criteria of the QCD sum rules are all satisfied, and we expect to make reliable predictions.

In Table V, we present the possible assignments of the ground state hidden-charm tetraquark states. There are one scalar tetraquark candidate with the spin-parity-chargeconjugation $J^{P C}=0^{++}$for the $X(3860)$, one scalar tetraquark candidate with the $J^{P C}=0^{++}$for the $X(3915)$, one axialvector tetraquark candidate with the $J^{P C}=1^{++}$for the $X(3872)$, one axialvector tetraquark candidate with the $J^{P C}=1^{+-}$for the $Z_{c}(3900)$, one axialvector tetraquark candidate with the $J^{P C}=1^{+-}$for the $Z_{c}(4600)$, three axialvector tetraquark candidates with the $J^{P C}=1^{+-}$for the $Z_{c}(4020)$ and $Z_{c}(4055)$, two axialvector tetraquark candidates with the $J^{P C}=1^{++}$for the $Z_{c}(4050)$, and one tensor tetraquark candidate with the $J^{P C}=2^{++}$for the $Z_{c}(4050)$.

In 2008, the Belle Collaboration observed two resonancelike structures, which are now known as the $Z_{c}(4050)$ and $Z_{c}(4250)$, in the $\pi^{+} \chi_{c 1}$ mass spectrum in the decays

TABLE V. The possible assignments of the ground state hidden-charm tetraquark states; the isospin limit is implied.

\begin{tabular}{|c|c|c|c|c|}
\hline$Z_{c}\left(X_{c}\right)$ & $J^{P C}$ & $M_{Z}(\mathrm{GeV})$ & Assignments & $Z_{c}^{\prime}\left(X_{c}^{\prime}\right)$ \\
\hline$[u c]_{S}[\overline{d c}]_{S}$ & $0^{++}$ & $3.88 \pm 0.09$ & $? X(3860)$ & \\
\hline$[u c]_{A}[\overline{d c}]_{A}$ & $0^{++}$ & $3.95 \pm 0.09$ & $? X(3915)$ & \\
\hline$[u c]_{\tilde{A}}[\overline{d c}]_{\tilde{A}}$ & $0^{++}$ & $3.98 \pm 0.08$ & & \\
\hline$[u c]_{V}[\overline{d c}]_{V}$ & $0^{++}$ & $4.65 \pm 0.09$ & & \\
\hline$[u c]_{\tilde{V}}[\overline{d c}]_{\tilde{V}}$ & $0^{++}$ & $5.35 \pm 0.09$ & & \\
\hline$[u c]_{P}[\overline{d c}]_{P}$ & $0^{++}$ & $5.49 \pm 0.09$ & & \\
\hline$[u c]_{S}[\overline{d c}]_{A}-[u c]_{A}[\overline{d c}]_{S}$ & $1^{+-}$ & $3.90 \pm 0.08$ & $? Z_{c}(3900)$ & $? Z_{c}(4430)$ \\
\hline$[u c]_{A}[\overline{d c}]_{A}$ & $1^{+-}$ & $4.02 \pm 0.09$ & $? Z_{c}(4020 / 4055)$ & $? Z_{c}(4600)$ \\
\hline$[u c]_{S}[\overline{d c}]_{\tilde{A}}-[u c]_{\tilde{A}}[\overline{d c}]_{S}$ & $1^{+-}$ & $4.01 \pm 0.09$ & $? Z_{c}(4020 / 4055)$ & $? Z_{c}(4600)$ \\
\hline$[u c]_{\tilde{A}}[\overline{d c}]_{A}-[u c]_{A}[\overline{d c}]_{\tilde{A}}$ & $1^{+-}$ & $4.02 \pm 0.09$ & $? Z_{c}(4020 / 4055)$ & $? Z_{c}(4600)$ \\
\hline$[u c]_{\tilde{V}}[\overline{d c}]_{V}+[u c]_{V}[\overline{d c}]_{\tilde{V}}$ & $1^{+-}$ & $4.66 \pm 0.10$ & $? Z_{c}(4600)$ & \\
\hline$[u c]_{V}[\overline{d c}]_{V}$ & $1^{+-}$ & $5.46 \pm 0.09$ & & \\
\hline$[u c]_{P}[\overline{d c}]_{V}+[u c]_{V}[\overline{d c}]_{P}$ & $1^{+-}$ & $5.45 \pm 0.09$ & & \\
\hline$[u c]_{S}[\overline{d c}]_{A}+[u c]_{A}[\overline{d c}]_{S}$ & $1^{++}$ & $3.91 \pm 0.08$ & $? X(3872)$ & \\
\hline$[u c]_{S}[\overline{d c}]_{\tilde{A}}+[u c]_{\tilde{A}}[\overline{d c}]_{S}$ & $1^{++}$ & $4.02 \pm 0.09$ & $? Z_{c}(4050)$ & \\
\hline$[u c]_{\tilde{V}}[\overline{d c}]_{V}-[u c]_{V}[\overline{d c}]_{\tilde{V}}$ & $1^{++}$ & $4.08 \pm 0.09$ & $? Z_{c}(4050)$ & \\
\hline$[u c]_{\tilde{A}}[\overline{d c}]_{A}+[u c]_{A}[\overline{d c}]_{\tilde{A}}$ & $1^{++}$ & $5.19 \pm 0.09$ & & \\
\hline$[u c]_{P}[\overline{d c}]_{V}-[u c]_{V}[\overline{d c}]_{P}$ & $1^{++}$ & $5.46 \pm 0.09$ & & \\
\hline$[u c]_{A}[\overline{d c}]_{A}$ & $2^{++}$ & $4.08 \pm 0.09$ & $? Z_{c}(4050)$ & \\
\hline$[u c]_{V}[\overline{d c}]_{V}$ & $2^{++}$ & $5.40 \pm 0.09$ & & \\
\hline
\end{tabular}


$\bar{B}^{0} \rightarrow K^{-} \pi^{+} \chi_{c 1}$ with a statistical significance exceeds $5 \sigma$ [48]. In 2014, the Belle Collaboration observed evidence for the $Z_{c}(4055)$ in the $\pi^{ \pm} \psi^{\prime}$ mass spectrum in the decays $Y(4360) \rightarrow \pi^{+} \pi^{-} \psi^{\prime}$ with a statistical significance of $3.5 \sigma$ [49]. The $Z_{c}^{+}(4050), Z_{c}^{ \pm}(4055)$ and $Z_{c}^{+}(4250)$ have not been confirmed by other experiments yet.

The $Z_{c}^{+}(4050), Z_{c}^{ \pm}(4055)$, and $Z_{c}^{+}(4250)$ are not necessary to have the definite charge conjugation $C=+,-$, and + , respectively, as they are not the charge conjugation eigenstates. The possible quantum numbers of the $Z_{c}^{+}(4050)$ and $Z_{c}^{+}(4250)$ are the spin-parity $J^{P}=0^{+}, 1^{-}, 1^{+}$, and $2^{+}$. From Table $\mathrm{V}$, we can see that there is no candidate for the $Z_{c}^{+}(4250)$ in the case of the spin parity $\mathrm{J}^{P}=0^{+}, 1^{+}$, and $2^{+}$. In Ref. [21], we observe that the $Z_{c}(4250)$ can be assigned to be a vector tetraquark state with a relative $P$ wave between the diquark and antidiquark constituents based on the predictions of the QCD sum rules.

From Table V, we can see that the lowest tetraquark state with the $J^{P C}=1^{+-}$has a mass about $3.9 \mathrm{GeV}$. If the energy gap between the ground state and the first radial excited state is about $0.5-0.6 \mathrm{GeV}$, the first radial excitation of the axialvector tetraquark state has a mass about $4.4-4.5 \mathrm{GeV}$, which happens to coincide with the experimental mass of the $Z_{c}(4430)$; see Table I. The $Z_{c}(3900)$ and $Z_{c}(4430)$ can be assigned to be the ground state and the first radial excited axialvector tetraquark states with the $J^{P C}=1^{+-}$, respectively [43-45]; the QCD sum rules supports such assignments [45]. The QCD sum rules also support assigning the $Z_{c}(4600)$ to be the first radial excited state of the $Z_{c}(4020)$ [30,31].

The $Z_{c}(4200)$ has the spin-parity-charge-conjugation $J^{P C}=1^{+-}$; there is no room to accommodate it in the scenario of pure tetraquark state. The pure axialvector tetraquark states have masses of about $3.9 \mathrm{GeV}, 4.0 \mathrm{GeV}$, $4.7 \mathrm{GeV}$, and $5.5 \mathrm{GeV}$, respectively, which are inconsistent with the experimental value $M_{Z_{c}(4200)}=4196_{-29}^{+31+17} \mathrm{MeV}$ [2]. However, a mixing $[u c]_{S}[\overline{d c}]_{A}-[u c]_{A}[\overline{d c}]_{S}$ or $[u c]_{A}[\overline{d c}]_{A}$ or $[u c]_{S}[\overline{d c}]_{\tilde{A}}-[u c]_{\tilde{A}}[\overline{d c}]_{S}$ or $[u c]_{\tilde{A}}[\overline{d c}]_{A}-[u c]_{A}[\overline{d c}]_{\tilde{A}} \oplus$ $[u c]_{\tilde{V}}[\overline{d c}]_{V}+[u c]_{V}[\overline{d c}]_{\tilde{V}}$ or $[u c]_{V}[\overline{d c}]_{V}$ or $[u c]_{P}[\overline{d c}]_{V}+$ $[u c]_{V}[\overline{d c}]_{P}$ type axialvector tetraquark state with the spinparity-charge-conjugation $J^{P C}=1^{+-}$may be have a mass about $4.2 \mathrm{GeV}$. On the other hand, in Ref. [50], we observe that the $Z_{c}(4200)$ can be assigned to be the color-octet-color-octet $\left(\mathbf{8}_{c} \mathbf{8}_{c}\right)$ type tetraquark state by calculating the mass and width with the QCD sum rules.

If the $Z_{c}(4100)$ has the spin-parity-charge-conjugation $J^{P C}=0^{++}$, it cannot be a pure scalar tetraquark candidate according to the predictions presented in Table V. In Ref. [29], we observe that if we introduce the mixing effects, a mixing $[u c]_{\tilde{A}}[\overline{d c}]_{\tilde{A}} \oplus[u c]_{\tilde{V}}[\overline{d c}]_{\tilde{V}}$ type scalar tetraquark state can have a mass about $4.1 \mathrm{GeV}$. From Table V, we can see that a mixing $[u c]_{S}[\overline{d c}]_{S}$ or $[u c]_{A}[\overline{d c}]_{A}$ or $[u c]_{\tilde{A}}[\overline{d c}]_{\tilde{A}} \oplus[u c]_{V}[\overline{d c}]_{V}$ or $[u c]_{\tilde{V}}[\overline{d c}]_{\tilde{V}}$ or $[u c]_{P}[\overline{d c}]_{P}$ type scalar tetraquark state with suitable mixing angles can have a mass about $4.1 \mathrm{GeV}$.
On the other hand, in Refs. [21,22], we observe that the lowest vector tetraquark state from the QCD sum rules has a mass about $4.24 \pm 0.10 \mathrm{GeV}$, which lies above the $Z_{c}(4100)$; the $Z_{c}(4100)$ is unlikely to be a vector tetraquark state.

If we abandon the constraint $\mu=\sqrt{M_{X / Y / Z}^{2}-\left(2 M_{c}\right)^{2}}$ in choosing the suitable energy scales of the QCD spectral densities and assign the $Z_{c}(4200)$ to be the $[u c]_{S}[\overline{d c}]_{A}-$ $[u c]_{A}[\overline{d c}]_{S}$ type hidden-charm tetraquark state with the quantum numbers $J^{P}=1^{+-}$, we can reproduce the experimental value $M_{Z_{c}(4200)}=4196_{-29}^{+31+17} \mathrm{MeV}$ from the Belle Collaboration by choosing the energy scale $\mu=$ $1.2 \mathrm{GeV}$ [50]. Furthermore, if we assign the $Z_{c}(4100)$ to be the $[u c]_{\tilde{A}}[\overline{d c}]_{\tilde{A}}$ type hidden-charm tetraquark state with the quantum numbers $J^{P}=0^{++}$, we obtain the prediction $M_{Z_{c}(4100)}=4.12 \pm 0.08 \mathrm{GeV}$ by choosing the energy scale $\mu=1.4 \mathrm{GeV}$ [29], which is in excellent agreement with the experimental value $M_{Z_{c}}=4096 \pm 20_{-22}^{+18} \mathrm{MeV}$ from the LHCb Collaboration [3]. However, the energy scales $\mu=1.2 \mathrm{GeV}$ and $1.4 \mathrm{GeV}$ are chosen by hand and are inconsistent with the energy scales presented in Table III; furthermore, the two energy scales are even inconsistent with each other. Without introducing mixing effects among the tetraquark states, the QCD sum rules disfavor assigning the $Z_{c}(4100)$ and $Z_{c}(4200)$ to be the hidden-charm tetraquark states.

The $X(3940)$ and $X(4160)$ were observed in the processes $e^{+} e^{-} \rightarrow J / \psi D^{*} \bar{D}$ and $J / \psi D^{*} \bar{D}^{*}$, respectively, by the Belle Collaboration [51,52]. The absence of signals for any of the known nonzero spin charmonium states in the distribution of masses recoiling from the $J / \psi$ in the inclusive spectrum provides circumstantial evidence for zero spin assignments for the $X(3940)$ and $X(4160)$. If they have zero spin, the observed decays $X(3940) \rightarrow D^{*} \bar{D}$ and $X(4160) \rightarrow$ $D^{*} \bar{D}^{*}$ support that they have the quantum numbers $J^{P C}=0^{-+}$; however, the assignment of the $J^{P C}=0^{++}$ for the $X(4160)$ cannot be excluded. The calculations based on the QCD sum rules indicate that the tetraquark states with the $J^{P C}=0^{-+}$have much larger masses than the $X(4160)$; the numerical results will be presented elsewhere. On the other hand, if the $X(4160)$ is an scalar hidden-charm tetraquark state, we have to introduce the mixing effects to account for the mass. In summary, there are no rooms to accommodate the $X(3940)$ and $X(4160)$ in the scenario of tetraquark states without fine-tuning. They may be the conventional $\eta_{c}(3 \mathrm{~S})$ and $\eta_{c}(4 \mathrm{~S})$ with the quantum numbers $J^{P C}=0^{-+}$, respectively [53]. However, the measured masses lie far below expectations based on the potential models. More theoretical and experimental works are still needed to explore the nature of the $X(3940)$ and $X(4160)$.

The hidden-charm tetraquark masses obtained in the present work can be confronted to the experimental data at the BESIII, LHCb, Belle II, CEPC, FCC, ILC in the future and shed light on the nature of the exotic $X, Y, Z$ particles. 
Although the mass is the basic and most important parameter in describing a hadron, we cannot assign a hadron unambiguously based on the mass alone, we need other quantum numbers, such as the spin, parity, and conjugation, and have to study its productions and decays. For example, the $X(3872)$ can be assigned to be the tetraquark state or $\mathbf{1}_{c} \mathbf{1}_{c}$-type tetraquark state based on the predicted mass from the QCD sum rules; however, without introducing the $c \bar{c}$ or $\chi_{c 1}^{\prime}$ component, we cannot describe its productions at the hadron colliders and the hadronic decays $X(3872) \rightarrow$ $J / \psi \pi^{+} \pi^{-}$and $J / \psi \pi^{+} \pi^{-} \pi^{0}[54,55]$.

We can take the pole residues $\lambda_{Z}$ as the basic input parameters to study the two-body strong decays of those hidden-charm tetraquark states,

$$
\begin{aligned}
Z_{c}^{ \pm}\left(1^{+-}\right) & \rightarrow \pi^{ \pm} J / \psi, \pi^{ \pm} \psi^{\prime}, \pi^{ \pm} h_{c}, \rho^{ \pm} \eta_{c},\left(D \bar{D}^{*}\right)^{ \pm},\left(D^{*} \bar{D}\right)^{ \pm},\left(D^{*} \bar{D}^{*}\right)^{ \pm} \\
Z_{c}^{ \pm}\left(0^{++}\right) & \rightarrow \pi^{ \pm} \eta_{c}, \pi^{ \pm} \chi_{c 1}, \rho^{ \pm} J / \psi, \rho^{ \pm} \psi^{\prime},(D \bar{D})^{ \pm},\left(D^{*} \bar{D}^{*}\right)^{ \pm} \\
Z_{c}^{ \pm}\left(1^{++}\right) & \rightarrow \pi^{ \pm} \chi_{c 1}, \rho^{ \pm} J / \psi, \rho^{ \pm} \psi^{\prime},\left(D \bar{D}^{*}\right)^{ \pm},\left(D^{*} \bar{D}\right)^{ \pm},\left(D^{*} \bar{D}^{*}\right)^{ \pm} \\
Z_{c}^{ \pm}\left(2^{++}\right) & \rightarrow \pi^{ \pm} \eta_{c}, \pi^{ \pm} \chi_{c 1}, \rho^{ \pm} J / \psi, \rho^{ \pm} \psi^{\prime},(D \bar{D})^{ \pm},\left(D^{*} \bar{D}^{*}\right)^{ \pm} \\
Z_{c}^{0}\left(1^{+-}\right) & \rightarrow \pi^{0} J / \psi, \pi^{0} \psi^{\prime}, \pi^{0} h_{c}, \rho^{0} \eta_{c},\left(D \bar{D}^{*}\right)^{0},\left(D^{*} \bar{D}\right)^{0},\left(D^{*} \bar{D}^{*}\right)^{0}, \\
Z_{c}^{0}\left(0^{++}\right) & \rightarrow \pi^{0} \eta_{c}, \pi^{0} \chi_{c 1}, \rho^{0} J / \psi, \rho^{0} \psi^{\prime},(D \bar{D})^{0},\left(D^{*} \bar{D}^{*}\right)^{0} \\
Z_{c}^{0}\left(1^{++}\right) & \rightarrow \pi^{0} \chi_{c 1}, \rho^{0} J / \psi, \rho^{0} \psi^{\prime},\left(D \bar{D}^{*}\right)^{0},\left(D^{*} \bar{D}\right)^{0},\left(D^{*} \bar{D}^{*}\right)^{0} \\
Z_{c}^{0}\left(2^{++}\right) & \rightarrow \pi^{0} \eta_{c}, \pi^{0} \chi_{c 1}, \rho^{0} J / \psi, \rho^{0} \psi^{\prime},(D \bar{D})^{0},\left(D^{*} \bar{D}^{*}\right)^{0} \\
X\left(1^{+-}\right) & \rightarrow \eta J / \psi, \eta \psi^{\prime}, \eta h_{c}, \omega \eta_{c},(D \bar{D})^{*},\left(D^{*} \bar{D}\right)^{0},\left(D^{*} \bar{D}^{*}\right)^{0} \\
X\left(0^{++}\right) & \rightarrow \eta \eta_{c}, \eta \chi_{c 1}, \omega J / \psi, \omega \psi^{\prime},(D \bar{D})^{0},\left(D^{*} \bar{D}^{*}\right)^{0} \\
X\left(1^{++}\right) & \rightarrow \eta \chi_{c 1}, \omega J / \psi, \omega \psi^{\prime},\left(D \bar{D}^{*}\right)^{0},\left(D^{*} \bar{D}\right)^{0},\left(D^{*} \bar{D}^{*}\right)^{0} \\
X\left(2^{++}\right) & \rightarrow \eta \eta_{c}, \eta \chi_{c 1}, \omega J / \psi, \omega \psi^{\prime},(D \bar{D})^{0},\left(D^{*} \bar{D}^{*}\right)^{0}
\end{aligned}
$$

with the three-point QCD sum rules or the light cone QCD sum rules and obtain the partial decay widths to diagnose the nature of the $Z_{c}$ and $X$ states. For example, we tentatively assign the $Z_{c}^{+}(3900)$ to be the $[u c]_{S}[\overline{d c}]_{A}-$ $[u c]_{A}[\overline{d c}]_{S}$ type axialvector tetraquark state, study the twobody strong decays $Z_{c}^{+}(3900) \rightarrow J / \psi \pi^{+}, \eta_{c} \rho^{+}, D^{+} \bar{D}^{* 0}$, $\bar{D}^{0} D^{*+}$ with the QCD sum rules based on solid quarkhadron duality, and produce the experimental value of the total width [56]. Experimentally, the BESIII Collaboration measured the ratios of the partial widths of the decays $Z_{c}(3900 / 4020) \rightarrow \eta_{c} \rho, J / \psi \pi$ at the $90 \%$ C.L. [57].

\section{CONCLUSION}

In this article, we take the pseudoscalar, scalar, axialvector, vector, and tensor charmed (anti)diquark operators as the basic constituents and construct the scalar, axialvector, and tensor hidden-charm tetraquark currents to study the mass spectrum of the ground state hidden-charm tetraquark states with the QCD sum rules in a comprehensive way. In calculations, we carry out the operator product expansion up to the vacuum condensates of dimension 10 to obtain the QCD spectral densities and use the energy scale formula $\mu=\sqrt{M_{X / Y / Z}^{2}-\left(2 \mathbb{M}_{c}\right)^{2}}$ to determine the ideal energy scales. The present predictions support assigning the $X(3860)$ to be the $[q c]_{S}[\overline{q c}]_{S}$ type scalar tetraquark state with the $J^{P C}=0^{++}$, assigning the $X(3915)$ to be the $[q c]_{A}[\overline{q c}]_{A}$ type scalar tetraquark state with the $J^{P C}=0^{++}$, assigning the $X(3872)$ to be the $[q c]_{S}[\overline{q c}]_{A}+[q c]_{A}[\overline{q c}]_{S}$ type axialvector tetraquark state with the $J^{P C}=1^{++}$, assigning the $Z_{c}(3900)$ to be the $[u c]_{S}[\overline{d c}]_{A}-[u c]_{A}[\overline{d c}]_{S}$ type axialvector tetraquark state with the $J^{P C}=1^{+-}$, assigning the $Z_{c}(4020)$ and $Z_{c}(4055)$ to be the $[u c]_{A}[\overline{d c}]_{A}$ type, $[u c]_{S}[\overline{d c}]_{\tilde{A}}-[u c]_{\tilde{A}}[\overline{d c}]_{S}$ type or $[u c]_{\tilde{A}}[\overline{d c}]_{A}-[u c]_{A}[\overline{d c}]_{\tilde{A}}$ type axialvector tetraquark states with the $J^{P C}=1^{+-}$, assigning the $Z_{c}(4600)$ to be the $[u c]_{\tilde{V}}[\overline{d c}]_{V}+[u c]_{V}[\overline{d c}]_{\tilde{V}}$ type axialvector tetraquark state with the $J^{P C}=1^{+-}$, or the first radial excited state of the $Z_{c}(4020)$, assigning the $Z_{c}(4050)$ to be the $[u c]_{S}[\overline{d c}]_{\tilde{A}}+$ $[u c]_{\tilde{A}}[\overline{d c}]_{S}$ type or $[u c]_{\tilde{V}}[\overline{d c}]_{V}-[u c]_{V}[\overline{d c}]_{\tilde{V}}$ type axialvector tetraquark state with the $J^{P C}=1^{++}$or $[u c]_{A}[\overline{d c}]_{A}$ type tensor tetraquark state with the $J^{P C}=2^{++}$, and assigning the $Z_{c}$ (4430) to be the first radial excited state of the $Z_{c}(3900)$. More experimental data and theoretical work are still needed to make unambiguous assignments. There are no rooms to accommodate the $X(3940), X(4160), Z_{c}(4100)$, $Z_{c}(4200)$ in the scenario of tetraquark states without finetuning. The $X(3940)$ and $X(4160)$ may be the conventional $\eta_{c}(3 \mathrm{~S})$ and $\eta_{c}(4 \mathrm{~S})$ states with the $J^{P C}=0^{-+}$, respectively. While the $Z_{c}(4100)$ may be a mixing scalar tetraquark state with the $J^{P C}=0^{++}$, the $Z_{c}(4200)$ may be an axialvector 
color-octet-color-octet type tetraquark state with the $J^{P C}=1^{+-}$. The predicted tetraquark masses can be confronted to the experimental data in the future at the BESIII, LHCb, Belle II, CEPC, FCC, ILC.

\section{ACKNOWLEDGMENTS}

This work is supported by National Natural Science Foundation, Grant No. 11775079.
[1] S. K. Choi et al., Phys. Rev. Lett. 91, 262001 (2003).

[2] M. Tanabashi et al., Phys. Rev. D 98, 030001 (2018).

[3] R. Aaij et al., Eur. Phys. J. C 78, 1019 (2018).

[4] H. X. Chen, W. Chen, X. Liu, and S. L. Zhu, Phys. Rep. 639, 1 (2016).

[5] R. F. Lebed, R. E. Mitchell, and E. S. Swanson, Prog. Part. Nucl. Phys. 93, 143 (2017).

[6] A. Esposito, A. Pilloni, and A. D. Polosa, Phys. Rep. 668, 1 (2017).

[7] F. K. Guo, C. Hanhart, U. G. Meissner, Q. Wang, Q. Zhao, and B. S. Zou, Rev. Mod. Phys. 90, 015004 (2018).

[8] A. Ali, J. S. Lange, and S. Stone, Prog. Part. Nucl. Phys. 97, 123 (2017).

[9] S. L. Olsen, T. Skwarnicki, and D. Zieminska, Rev. Mod. Phys. 90, 015003 (2018).

[10] R. M. Albuquerque, J. M. Dias, K. P. Khemchandani, A. M. Torres, F. S. Navarra, M. Nielsen, and C. M. Zanetti, J. Phys. G 46, 093002 (2019).

[11] Y. R. Liu, H. X. Chen, W. Chen, X. Liu, and S. L. Zhu, Prog. Part. Nucl. Phys. 107, 237 (2019).

[12] N. Brambilla, S. Eidelman, C. Hanhart, A. Nefediev, C. P. Shen, C. E. Thomas, A. Vairo, and C. Z. Yuan, arXiv:1907.07583.

[13] M. A. Shifman, A. I. Vainshtein, and V. I. Zakharov, Nucl. Phys. B147, 385 (1979); B147, 448 (1979).

[14] L. J. Reinders, H. Rubinstein, and S. Yazaki, Phys. Rep. 127, 1 (1985).

[15] P. Colangelo and A. Khodjamirian, Qcd Sum Rules, A Modern Perspective, At the Frontier of Particle Physics (World Scientific, Singapore, 2001), pp. 1495-1576.

[16] R. D. Matheus, S. Narison, M. Nielsen, and J. M. Richard, Phys. Rev. D 75, 014005 (2007).

[17] Z. G. Wang and T. Huang, Phys. Rev. D 89, 054019 (2014).

[18] J. R. Zhang and M. Q. Huang, Phys. Rev. D 83, 036005 (2011); J. R. Zhang, Phys. Rev. D 87, 116004 (2013).

[19] Z. G. Wang, Eur. Phys. J. C 74, 2874 (2014).

[20] Z. G. Wang and T. Huang, Nucl. Phys. A930, 63 (2014); Z. G. Wang, Eur. Phys. J. C 79, 489 (2019).

[21] Z. G. Wang, Eur. Phys. J. C 78, 933 (2018); 79, 29 (2019).

[22] Z. G. Wang, Eur. Phys. J. C 78, 518 (2018); 79, 184 (2019).

[23] C. F. Qiao and L. Tang, Eur. Phys. J. C 74, 2810 (2014).

[24] S. S. Agaev, K. Azizi, and H. Sundu, Phys. Rev. D 93, 074002 (2016); H. Sundu, S. S. Agaev, and K. Azizi, Eur. Phys. J. C 79, 215 (2019).

[25] W. Chen and S. L. Zhu, Phys. Rev. D 83, 034010 (2011).
[26] Z. G. Wang, Eur. Phys. J. C 77, 78 (2017); Eur. Phys. J. A 53, 19 (2017).

[27] Z. G. Wang and Z. Y. Di, Eur. Phys. J. C 79, 72 (2019); Z. G. Wang, Acta Phys. Pol. B 51, 435 (2020).

[28] R. F. Lebed and A. D. Polosa, Phys. Rev. D 93, 094024 (2016).

[29] Z. G. Wang, Commun. Theor. Phys. 71, 1319 (2019).

[30] H. X. Chen and W. Chen, Phys. Rev. D 99, 074022 (2019).

[31] Z. G. Wang, Chin. Phys. C 44, 063105 (2020).

[32] A. Selem and F. Wilczek, Hadron Systematics and Emergent Diquarks, New Trends in HERA Physics 2005 (World Scientific, Singapore, 2006), pp. 337-356.

[33] L. Maiani, A. D. Polosa, and V. Riquer, Phys. Lett. B 778, 247 (2018).

[34] L. Maiani, A. D. Polosa, and V. Riquer, Phys. Rev. D 100, 014002 (2019).

[35] S. J. Brodsky, D. S. Hwang, and R. F. Lebed, Phys. Rev. Lett. 113, 112001 (2014).

[36] W. Lucha, D. Melikhov, and H. Sazdjian, Phys. Rev. D 100, 014010 (2019); 100, 074029 (2019).

[37] Z. G. Wang, Phys. Rev. D 101, 074011 (2020).

[38] L. D. Landau, Nucl. Phys. 13, 181 (1959).

[39] Z. G. Wang, arXiv:1910.09981.

[40] S. Narison and R. Tarrach, Phys. Lett. 125B, 217 (1983).

[41] Z. G. Wang, Eur. Phys. J. C 76, 387 (2016).

[42] B. L. Ioffe, Nucl. Phys. B188, 317 (1981).

[43] L. Maiani, F. Piccinini, A. D. Polosa, and V. Riquer, Phys. Rev. D 89, 114010 (2014).

[44] M. Nielsen and F. S. Navarra, Mod. Phys. Lett. A 29, 1430005 (2014).

[45] Z. G. Wang, Commun. Theor. Phys. 63, 325 (2015).

[46] R. Aaij et al., Phys. Rev. Lett. 122, 152002 (2019).

[47] Z. G. Wang, Int. J. Mod. Phys. A 34, 1950110 (2019).

[48] R. Mizuk et al., Phys. Rev. D 78, 072004 (2008).

[49] X. L. Wang et al., Phys. Rev. D 91, 112007 (2015).

[50] Z. G. Wang, Int. J. Mod. Phys. A 30, 1550168 (2015).

[51] K. Abe et al., Phys. Rev. Lett. 98, 082001 (2007).

[52] P. Pakhlov et al., Phys. Rev. Lett. 100, 202001 (2008).

[53] J. L. Rosner, AIP Conf. Proc. 815, 218 (2006); K. T. Chao, Phys. Lett. B 661, 348 (2008).

[54] C. Meng, H. Han, and K. T. Chao, Phys. Rev. D 96, 074014 (2017).

[55] R. D. Matheus, F. S. Navarra, M. Nielsen, and C. M. Zanetti, Phys. Rev. D 80, 056002 (2009).

[56] Z. G. Wang and J. X. Zhang, Eur. Phys. J. C 78, 14 (2018).

[57] C. Z. Yuan, Int. J. Mod. Phys. A 33, 1830018 (2018). 